\title{
Origins space telescope: from first light to life
}

\author{
M. C. Wiedner ${ }^{1}$ (D) S. Aalto ${ }^{2} \cdot$ L. Armus ${ }^{3} \cdot$ E. Bergin ${ }^{4} \cdot$ J. Birkby $^{5}$.

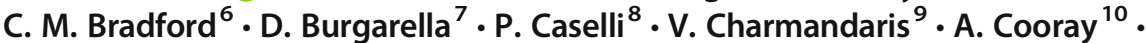 \\ E. De Beck ${ }^{2}$ • J. M. Desert ${ }^{5}$ - M. Gerin ${ }^{1}$ • J. Goicoechea ${ }^{11}$ - M. Griffin ${ }^{12}$. \\ P. Hartogh ${ }^{13}$ - F. Helmich ${ }^{14}$ - M. Hogerheijde ${ }^{15}$ - L. Hunt ${ }^{16}$ - A. Karska ${ }^{17}$. \\ Q. Kral ${ }^{18}$ - D. Leisawitz ${ }^{19}$ - G. Melnick ${ }^{20}$ - M. Meixner ${ }^{19,21}$ • M. Matsuura ${ }^{12}$. \\ S. Milam ${ }^{19}$ - C. Pearson ${ }^{22}$ - D. W. Pesce 20 . K. M. Pontoppidan ${ }^{21} \cdot$ A. Pope Po $^{23}$. \\ D. Rigopoulou ${ }^{24} \cdot$ T. Roellig ${ }^{25} \cdot$ I. Sakon ${ }^{26} \cdot$ J. Staguhn $^{19} \cdot$ K. Stevenson ${ }^{21}$
}

Received: 4 March 2021 / Accepted: 31 March 2021 / Published online: 13 September 2021

(C) The Author(s) 2021

\begin{abstract}
The Origins Space Telescope (Origins) is one of four science and technology definition studies selected by the National Aeronautics and Space Administration (NASA) in preparation of the 2020 Astronomy and Astrophysics Decadal survey in the US. Origins will trace the history of our origins from the time dust and heavy elements permanently altered the cosmic landscape to present-day life. It is designed to answer three major science questions: How do galaxies form stars, make metals, and grow their central supermassive black holes from reionization? How do the conditions for habitability develop during the process of planet formation? Do planets orbiting M-dwarf stars support life? Origins operates at mid- to far-infrared wavelengths from $\sim 2.8 \mu \mathrm{m}$ to $588 \mu \mathrm{m}$, and is more than 1000 times more sensitive than prior far-IR missions due to its cold $(\sim 4.5 \mathrm{~K})$ aperture and state-of-the-art instruments.
\end{abstract}

Keywords Spaceborn astrophysics $\cdot$ Mm-and sub-mm astronomy $\cdot$ Galaxy evolution $\cdot$ Star and planet formation $\cdot$ Exoplanets $\cdot$ ESA Voyage 2050

\footnotetext{
The Origins Space Telescope is a study commissioned by NASA and carried out by researchers, industry and NASA staff and has been summarized in the Origins Space Telescope Concept Study Report. The study has also been published in about 15 papers in the Journal of Astronomical Telescopes, Instruments and Systems (JATIS). This White Paper, submitted in response to the ESA Voyage 2050 Call, is based on the Origins study and reuses some figures and tables from the Study Report and the JATIS papers, in particular from Meixner et al. [15] , Leisawitz et al. [14] and Wiedner et al. [26], all of which were published under Creative Commons (CC BY 4.0) Gold Open Access (https://creativecommons.org/).
}

M. C. Wiedner

martina.wiedner@obspm.fr

A. Cooray

accoray@uci.edu

Extended author information available on the last page of the article 


\section{Introduction}

The Origins Space Telescope (Origins) will trace the history of our origins from the time dust and heavy elements permanently altered the cosmic landscape to present-day life. Origins operates at mid- to far-infrared wavelengths from $\sim 2.8 \mu \mathrm{m}$ to $588 \mu \mathrm{m}$, is more than 1000 times more sensitive than prior far-IR missions due to its cold $(\sim 4.5 \mathrm{~K})$ aperture and state-of-the-art instruments.

The Origins Space Telescope (Origins) ${ }^{12}$ investigates the creation and dispersal of elements essential to life, the formation of planetary systems, the transport of water to habitable worlds, and the atmospheres of exoplanets around nearby M-dwarfs to identify potentially habitable worlds. These science themes are motivated by their profound significance, as well as expected advances from, and limitations of, current and next-generation observatories (JWST, the Roman Space Telescope (previously known as WFIRST), ALMA, and the Rubin Observatory (previously known as LSST). The nine key Origins scientific objectives (Table 1) address NASA's three major astrophysics science goals: How does the Universe work?, How did we get here, and Are we alone? These nine aims also drive the instrumental requirements summarized in Table 2. The Origins design is powerful and versatile, and the infrared radiation it detects is information-rich. Origins will enable astronomers in the 2030s to ask new questions not yet imagined, and provide a far-infrared window complementary to planned, next-generation observatories (e.g., Athena, LISA, and ground-based ELTs).

The Origins Space Telescope (Origins) is one of four science and technology definition studies selected by NASA in preparation of the 2020 Astronomy and Astrophysics Decadal survey in the US. The study team included non-voting international representatives from ESA, JAXA, individual European countries, and Canada that contributed to the scientific and technical definition of Origins. A largely European team under French/CNES leadership designed the HEterodyne Receiver for Origins (HERO), which is one of the two upscope instruments.

The full Origins Mission Concept Study Report can be found at:

https://asd.gsfc.nasa.gov/firs/docs/

NASA has submitted four mission studies (LUVOIR, HabEX, Origins, and Lynx) to the Decadal survey and a prioritization is expected in 2021. If selected, Origins will begin Phase A in 2025 with a schedule that calls for a launch around 2035. This paper was submitted as a White Paper to the ESA Voyage 2050 call and describes the Origins Space Telescope to encourage ESA participation.

\footnotetext{
${ }^{1}$ https://origins.ipac.caltech.edu/

${ }^{2}$ https://asd.gsfc.nasa.gov/firs/
} 
Table 1 Scientific objectives for the Origins Space Telescope Table adapted from Fig. 1 of Leisawitz et al. [14]

\begin{tabular}{|c|c|c|c|}
\hline NASA Goal & How does the Universe work? & How did we get here? & Are we alone? \\
\hline $\begin{array}{l}\text { Origins Science } \\
\text { Goals }\end{array}$ & $\begin{array}{l}\text { How do galaxies } \\
\text { form stars, make } \\
\text { metals, and grow } \\
\text { their central } \\
\text { supermassive } \\
\text { black holes from } \\
\text { reionization to } \\
\text { today? }\end{array}$ & $\begin{array}{l}\text { How do the } \\
\text { conditions for } \\
\text { habitability } \\
\text { develop during } \\
\text { the process of } \\
\text { planet } \\
\text { formation? }\end{array}$ & $\begin{array}{l}\text { Do planets } \\
\text { orbiting M- } \\
\text { dwarf stars } \\
\text { support life? }\end{array}$ \\
\hline $\begin{array}{l}\text { Origins Scientific } \\
\text { Capabilities }\end{array}$ & $\begin{array}{l}\text { Origins will spectroscopically 3D } \\
\text { map wide extragalactic fields to } \\
\text { simultaneously measure properties } \\
\text { of growing supermassive black } \\
\text { holes and their galaxy hosts across } \\
\text { cosmic time. }\end{array}$ & $\begin{array}{l}\text { With sensitive, high-resolution } \\
\text { spectroscopy, Origins maps the } \\
\text { water trail from protoplanetary } \\
\text { discs to habitable worlds. }\end{array}$ & $\begin{array}{l}\text { By obtaining precise mid-infrared } \\
\text { transmission and emission } \\
\text { spectra, Origins will assess the } \\
\text { habitability of nearby exoplanets } \\
\text { and search for signs of life. }\end{array}$ \\
\hline $\begin{array}{l}\text { Origins } \\
\text { Scientific } \\
\text { Objectives }\end{array}$ & $\begin{array}{l}\text { 1) How does the relative growth of } \\
\text { stars and supermassive black } \\
\text { holes in galaxies evolve with time? } \\
\text { 2) How do galaxies make metals, } \\
\text { dust, and organic molecules? } \\
\text { 3) How do the relative energetics } \\
\text { from supernovae and quasars } \\
\text { influence the interstellar medium of } \\
\text { galaxies? }\end{array}$ & $\begin{array}{l}\text { 1) What role does water play in } \\
\text { the formation and evolution of } \\
\text { habitable planets? } \\
\text { 2) How and when do planets } \\
\text { form? } \\
\text { 3) How were water and life's } \\
\text { ingredients delivered to Earth and } \\
\text { to exoplanets? }\end{array}$ & $\begin{array}{l}\text { 1) What fraction of terrestrial } \\
\text { planets around K- and M-dwarf } \\
\text { stars has tenuous, clear, or } \\
\text { cloudy atmospheres? } \\
\text { 2) What fraction of terrestrial M- } \\
\text { dwarf planets is temperate? } \\
\text { 3) What types of temperate, } \\
\text { terrestrial, M-dwarf planets } \\
\text { support life? }\end{array}$ \\
\hline
\end{tabular}

\section{Key science goals and objectives}

\subsection{How do galaxies form stars, make metals, and grow their central supermassive black holes from reionization to today?}

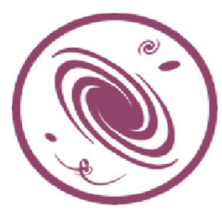

Origins is designed to answer these fundamental questions in galaxy formation and evolution through wide area spectral mapping surveys in the far-infrared (FIR) wavelengths. Origins is capable of carrying out 3D infrared spectral mapping surveys resulting in spectroscopic data on millions of galaxies spanning the redshift range of $z=0$ to $z>6$. These statistics are at a level comparable to the Sloan Digital Sky Survey (SDSS), but will be attained by Origins in a $2000 \mathrm{~h}$ survey, instead of $\sim 5$ years from the ground in the optical that took to complete SDSS. Moreover, the FIR regime probes highly obscured environments that are more prevalent at higher redshift.

A complete understanding of the astrophysical processes responsible for the formation and evolution of galaxies is one of the key scientific goals of modernday astrophysics. While we have made significant strides, there are still huge gaps 
Table 2 Science drives Origins key design parameters (Scientific Traceability Matrix (STM) provided in the full report) (Table adapted from Fig. 2 in Meixner et al. [15]

\begin{tabular}{|c|c|c|c|c|c|}
\hline \multicolumn{2}{|c|}{ Origins science driver } & \multicolumn{4}{|c|}{ Technical or instrument parameter } \\
\hline Scientific goal & Observable & Parameter & $\begin{array}{l}\text { Require- } \\
\text { ment }\end{array}$ & Design & Rationale \\
\hline \multirow{2}{*}{$\begin{array}{l}\text { How do } \\
\text { galaxie } \\
\text { s form } \\
\text { stars, make metals, } \\
\text { and grow their } \\
\text { central SMBHs? }\end{array}$} & \multirow{2}{*}{$\begin{array}{l}\text { Mid- and far-IR rest- } \\
\text { frame spectral lines. }\end{array}$} & $\begin{array}{l}\text { Telescope } \\
\text { Size }\end{array}$ & $3.0-5.0 \mathrm{~m}$ & $5.9 \mathrm{~m}$ & $\begin{array}{l}5.0 \mathrm{~m} \text { aperture is driven by the sensitivity to detect } z>6 \\
\text { galaxies, }>3.0 \mathrm{~m} \text { based on the sensitivity needed to detect } \\
\mathrm{z}>2 \text { galaxies. }\end{array}$ \\
\hline & & $\begin{array}{l}\text { Telescope } \\
\text { Temperatur } \\
\text { e }\end{array}$ & $<6 \mathrm{~K}$ & $4.5 \mathrm{~K}$ & $\begin{array}{l}\text { Sufficiently cold temperature to meet the sensitivity } \\
\text { requirements at the longest wavelengths, } T_{\text {tel }}>6 \mathrm{~K} \text { impacts } \\
\text { spectral line sensitivity at } \lambda>350 \mu \mathrm{m} \text {. }\end{array}$ \\
\hline \multirow{4}{*}{$\begin{array}{l}\text { condition } \\
\text { s for habitability } \\
\text { develop during the } \\
\text { process of planet } \\
\text { formation? }\end{array}$} & $\begin{array}{l}\mathrm{H}_{2}{ }^{18} \mathrm{O} \text { ground state } \\
547.4 \mu \mathrm{m}\end{array}$ & $\lambda_{\max }$ & $>550 \mu \mathrm{m}$ & $588 \mu \mathrm{m}$ & $\begin{array}{l}\mathrm{H}_{2}{ }^{18} \mathrm{O} \text { ground state line and the need to measure } \\
\text { continuum around it, } \lambda<500 \mu \mathrm{m} \text { impacts extragalactic } \\
\text { sciences. }\end{array}$ \\
\hline & $\mathrm{H}_{2}{ }^{18} \mathrm{O} 179.5-\mu \mathrm{m}$ line & $R=\lambda / \Delta \lambda$ & 200,000 & 203,000 & $\begin{array}{l}\text { Spectral resolving power is needed for Doppler tomography } \\
\text { to connect water emission lines to disc location. }\end{array}$ \\
\hline & \multirow[t]{2}{*}{$\begin{array}{c}\mathrm{HD} \\
112-\mu \mathrm{m} \text { line }\end{array}$} & $\begin{array}{l}\text { Spectral } \\
\text { line } \\
\text { sensitivity }\end{array}$ & $\begin{array}{l}10^{-20} \mathrm{~W} \mathrm{~m}^{-2} \\
(1 \mathrm{hr}, 5 \sigma)\end{array}$ & $\begin{array}{c}5 \times 10^{-21} \mathrm{~W} \\
\mathrm{~m}^{-2} \\
(1 \mathrm{hr}, 5 \sigma)\end{array}$ & $\begin{array}{l}\text { This sensitivity is required to measure disc gas masses and } \\
\text { obtain a useful sample of the population of discs at the } \\
\text { distance of Orion. }\end{array}$ \\
\hline & & $R=\lambda / \Delta \lambda$ & 40,000 & 43,000 & $\begin{array}{l}\text { The spectral resolving power needed for accurate gas } \\
\text { mass measurements. }\end{array}$ \\
\hline \multirow[b]{2}{*}{$\begin{array}{l}\text { Do } \\
\text { planets } \\
\text { orbiting } \\
\text { dwarf stars } \\
\text { life? }\end{array}$} & \multirow{2}{*}{$\begin{array}{l}\mathrm{CH}_{4}(3.3,7.4 \mu \mathrm{m}) \\
\mathrm{N}_{2} \mathrm{O}(4.5,7.8 \mu \mathrm{m}) \\
\mathrm{O}_{3}(9.7 \mu \mathrm{m}), \mathrm{CO}_{2} \\
(4.3,15 \mu \mathrm{m}), \mathrm{H}_{2}{ }^{18} \mathrm{O} \\
(6.3,17+\mu \mathrm{m})\end{array}$} & $\lambda_{\min }$ & $<3 \mu \mathrm{m}$ & $2.8 \mu \mathrm{m}$ & $\begin{array}{l}\mathrm{CO}_{2} \text { at } 4.3 \mu \mathrm{m} \text { is the strongest of all features, } \lambda_{\min }>5 \mu \mathrm{m} \\
\text { reduces the exoplanet case to surface temperature only. }\end{array}$ \\
\hline & & $\begin{array}{l}\text { Aperture } \\
\text { Size }\end{array}$ & $5.3 \mathrm{~m}$ & $5.9 \mathrm{~m}$ & $\begin{array}{l}\text { Aperture size determines the sensitivity to detect faint } \mathrm{CH}_{4} \\
\text { and } \mathrm{N}_{2} \mathrm{O} \text { lines, crucial for biosignature detection in } \\
\text { exoplanet transits over a } 5 \text {-year mission. }\end{array}$ \\
\hline
\end{tabular}

in our understanding of galaxy formation and evolution, especially the detailed astrophysical processes that grew and shaped galaxies over cosmic time. In particular, most of the accretion history of the Universe, both for star formation and for supermassive black hole (SMBH) growth, took place in highly obscured environments. The FIR wavelength regime traces physical processes even in such extreme conditions, whose importance grows with increasing redshift. While small targeted surveys are capable of solving some key problems, uncertainties related to our models of galaxy formation are still strongly tied to small number statistics of galaxies at high redshifts and biases coming from galaxy selections at various wavelengths that are either sensitive to older stellar populations, such as in the near-IR, or active galactic nuclei (AGN) activity, such as in the X-rays. At far-IR wavelengths, spectral lines trace all key ingredients of galaxies providing multifaceted probes of internal processes in play in galaxies (Fig. 1). Origins' wavelength range will not be explored by JWST, which is poised to provide the most detailed look yet at the distant Universe. Furthermore, with a sensitivity that is a factor of 1000 improvement over Spitzer and Herschel, Origins capability moves beyond simply detecting rare "tip-of-the-iceberg" dusty, starbursting galaxies above the stellar mass vs. star-formation rate main sequence to studying dust, gas and AGN in the dominant galaxy populations. Finally, with 3D spectral mapping surveys, Origins overcomes issues related to source confusion that impacted previous continuum mapping surveys with Herschel.

How do the stars and supermassive black holes in galaxies evolve with time? Origins allows us to peer through the obscuring dust, probe the physics of starformation through atomic and molecular gas, study the build-up of metals from dying stars, and establish the role of supermassive black holes (SMBHs) as they accrete and drive energetic outflows into the surrounding interstellar medium (Fig. 2). Key spectral signatures from the physical processes that sculpt galaxies are prominent in the infrared, where emission and absorption lines 
Fig. 1 Origins complements JWST mid-IR and ALMA sub$\mathrm{mm} / \mathrm{mm}$-wave capabilities. Origins will provide spectral line diagnostics indicative of AGN (red), star formation (blue), and feedback (green) over a wide range in redshifts, filling in a largely untapped region of wavelength and discovery space between JWST and ALMA. (Figure reproduced from Fig. 1 in Meixner et al. [15]

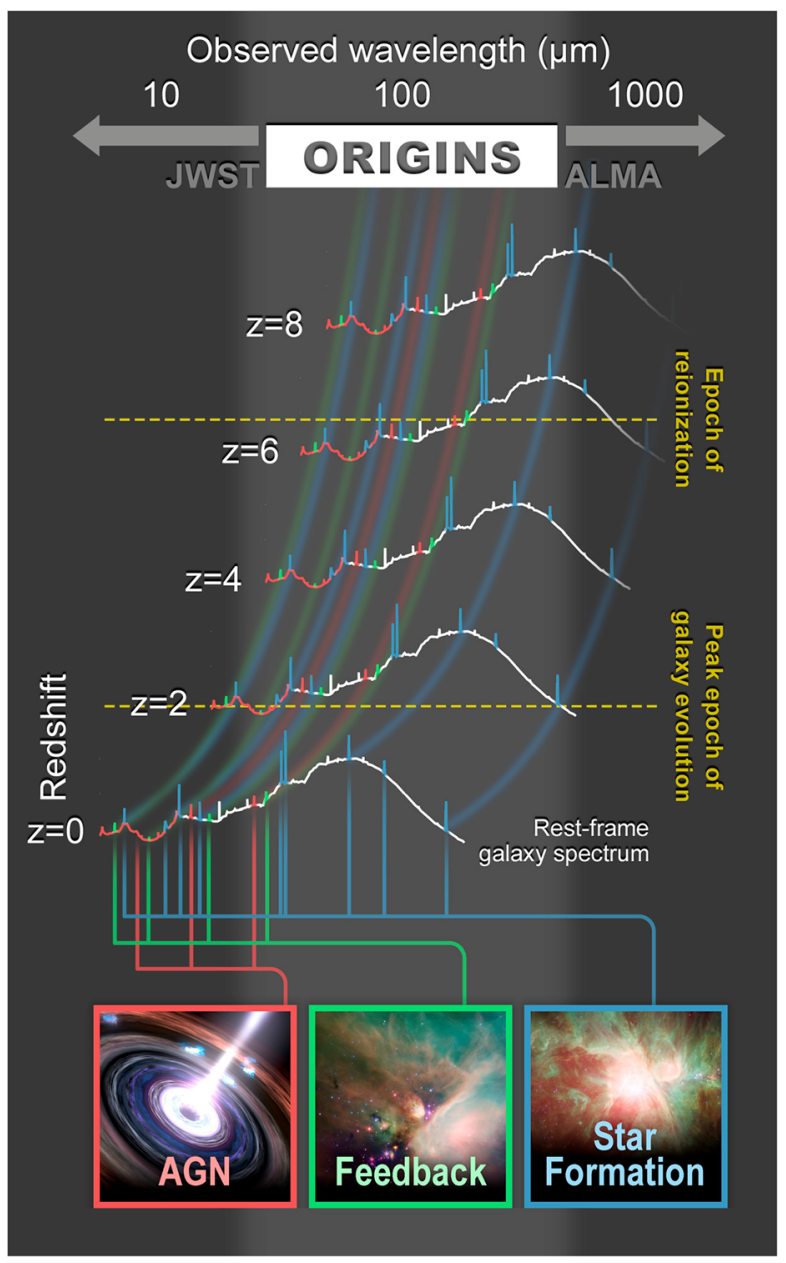

trace complex molecules, small and large dust grains, and atoms that are sensitive to changes in ionization and density [19].

How do galaxies make metals, dust, and organic molecules? Galaxies are the metal factories of the Universe, and Origins studies how metals and dust are made and dispersed throughout the cosmic web over the past 12 billion years. Sensitive metallicity indicators in the infrared can be used to track the growth history of elements via nucleosynthesis, even in the densest optically-obscured regions inside young galaxies at high redshift (metals: [23], dust: [21].

How do the relative energetics from supernovae and quasars influence the interstellar medium of galaxies? Galaxies are made of billions of stars, yet star formation is extremely inefficient on all scales, from single molecular clouds to galaxy clusters. Because of its power to penetrate obscuration, Origins can study the role of feedback processes at play in galaxies over a wide range of environments and redshifts (Fig. 1). Origins can study the processes that drive powerful outflows and map the demographics of galactic feedback [1] White Paper). 


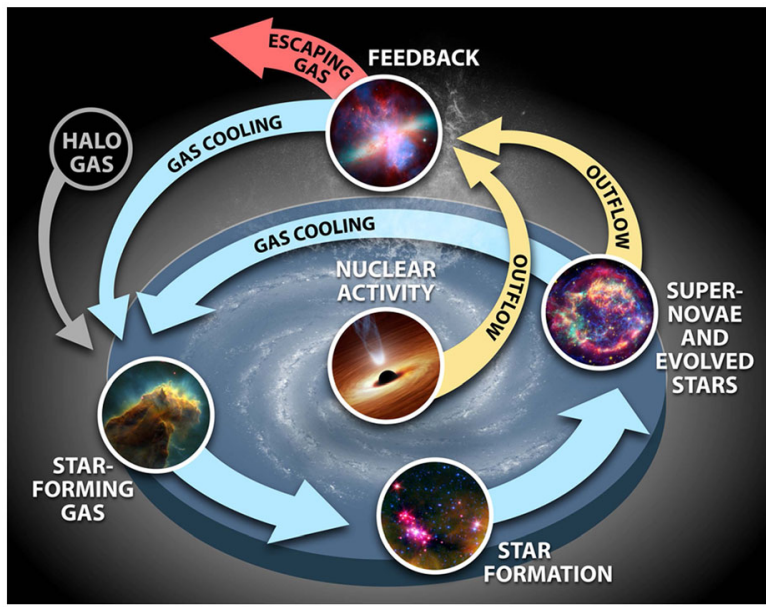

Fig. 2 Origins studies the baryon cycle in galaxies. Energetic processes that shape galaxies and the circumgalactic medium together define the galactic ecosystem. Through its ability to measure the energetics and dynamics of the atomic and molecular gas and dust in and around galaxies that are actively star-forming or have AGN activity, Origins can probe nearly all aspects of the galactic ecosystem: star formation and AGN growth, stellar death, AGN- and starburst-driven outflows, and gas cooling along with accretion. These measurements will provide a complete picture of the lifecycle of galaxies. (Figure reproduced from Fig. 2 in Meixner et al. [15]

\subsection{How do the conditions for habitability develop during the process of planet formation?}

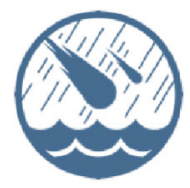

Water is essential for all life on our planet. Water provides the liquid medium for life's chemistry, while also playing an essential biochemical role. The formation story of water begins with the dynamical events, such as supernova explosions that gather and compress gas in the Interstellar Medium (ISM) and dust creating a latticework of filamentary clouds. It is within these dense clouds that stars and planets are born. Based on decades of study, we also know that water molecules formed as ice before stars are born in these dense clouds.

The Trail of Water begins as primordial interstellar material is provided to the young disc that will go on to form planets within tens of million years. A rotating collapsing cloud of gas and dust forms a young protostar surrounded by a disc that accretes material from a surrounding envelope. Within these proto-planetary discs, pebble-sized particles self-assemble under gravity to eventually form Earth-like worlds. Thanks to Herschel and Spitzer, and now with Atacama Large Millimeter Array (ALMA), we are able to construct the story of how these pre-planetary pebbles form and incorporate key molecules, such as water as ice from the natal cloud. However, without the ability to directly observe water in the forming disc, which Origins uniquely will provide, we cannot fully investigate this process. 


\subsubsection{What role does water play in the formation and evolution of habitable planets?}

The broad wavelength coverage offered by Origins includes a large number of highly useful water vapor transitions unavailable to any other telescope, including ALMA or JWST. In fact, with Origins we can study nearly two orders of magnitude more water lines than we can with either ALMA or JWST. Just as importantly, these water lines cover an astounding range in temperature, from the snow line to the steam line in discs (Fig. 3). With its unprecedented sensitivity to weak emission from less abundant forms of water, Origins provides the crucial measurement capability to understand water's role during a key evolutionary phase that lasts a few hundred thousand years and ends when the young star ablates and dissipates the surrounding natal cloud (see Fig. 4). Understanding the role of water in the initial phase of formation from ISM to discs is the basis for the first scientific objective in this key mission design science program (Table 1).

\subsubsection{How and when do planets form?}

During the subsequent phase that lasts a few million years when gas giants, such as Jupiter and Saturn, are born and the large Mars-sized embryos of Earth-like worlds are constructed, temperature plays a crucial role in determining what form of water will be found. If the environment is too hot, water will not exist as an ice but, instead, will be present as a vapor, if it is too cold, water will exist as an ice. Earth and the other terrestrial planets are constructed from coalescing solids. Over time, the newly formed star gradually dissipates the gaseous disc, ending the phase of gas giant formation. The remaining disc is filled with rocky bodies both large (Mars-sized) and small (asteroids). It is during this time, over tens of millions of years, that terrestrial worlds such as our own formed. However, key elements of this picture remain uncertain since the ice-line may migrate as the disc evolves and planets are born. Using the HD 1-0 $112 \mu \mathrm{m}$ line as a tracer of the gas content, the second objective in this key mission design science program will establish the total gas mass in proto-planetary discs down to a mass limit of one Neptune.

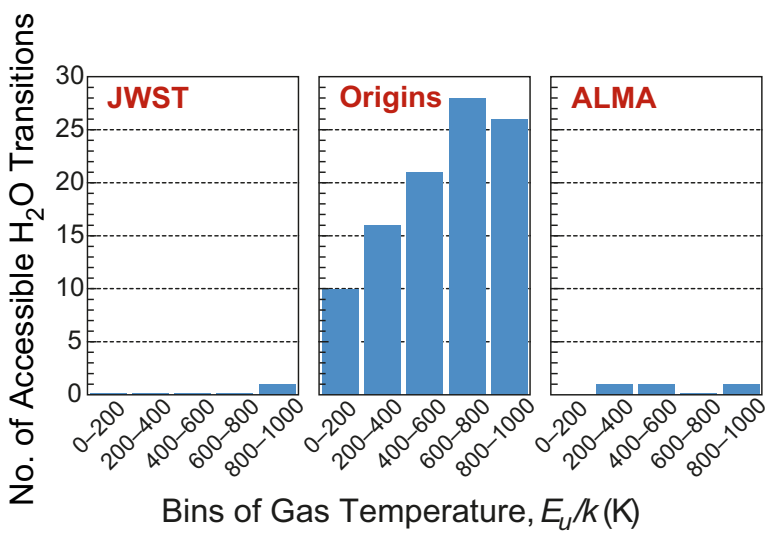

Fig. 3 Origins is capable of studying more than 100 transitions of water vapor, compared to one and three with JWST and ALMA, respectively. This plot shows the number of $\mathrm{H}_{2}{ }^{16} \mathrm{O}$ transitions observable by JWST, Origins, and ALMA as a function of the gas temperature, with energies above the ground state below $1000 \mathrm{~K}$. ALMA is limited by atmospheric absorption in its ability to observe water lines from the Galaxy. (Figure reproduced from Fig. 5 in Meixner et al. [15] 
Fig. 4 Origins will trace water and gas during all phases of the formation of a planetary system. The trail begins in the "pre-stellar" phase explored by Herschel, where a cloud of gas collapses (top) into a still-forming star surrounded by a disc nearly the size of our Solar System and a collapsing envelope of material (2nd from top). Over time, the envelope dissipates, leaving behind a young star and a disc with nascent planets ( 3 rd from top), eventually leaving behind a new planetary system (bottom). Origins will excel at probing the protoplanetary and later phases. (Figure reproduced from Fig. 3 in Meixner et al. [15]

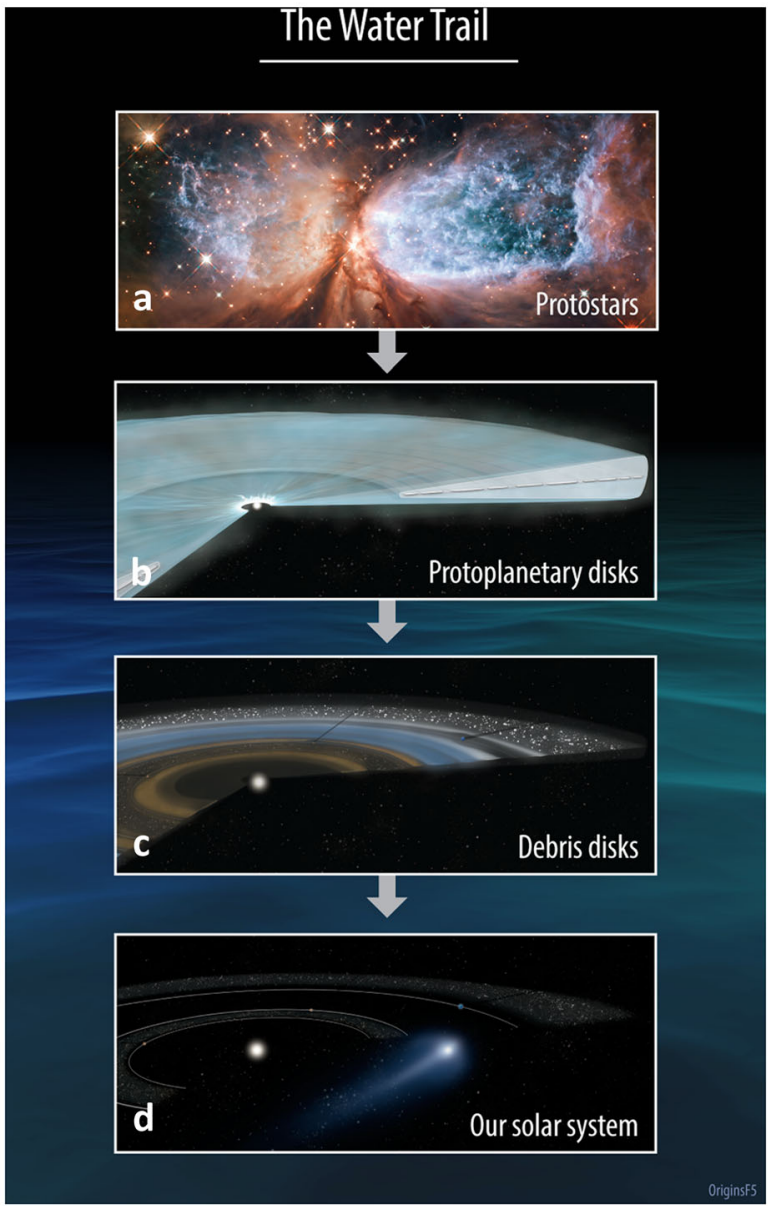

\subsubsection{How were water and life's ingredients delivered to Earth and to exoplanets?}

The dynamical interactions and the construction of rocky worlds by energetic impacts leads to a phase that generates significant "debris" from the collisions. The "ice-line" - the distance from the young star where water transitions from a gas to a solid - holds a prominent place in planet-formation, as it is believed that the Earth, and its precursor materials, formed inside our Solar System's ice-line. It is theorized that water was delivered to the early Earth via impacts from material that formed beyond the ice-line during this debris phase. While debris discs have been mapped using prior space telescopes, they did not provide the capability to determine whether the impactors carry water. Consistent with the picture of water delivery, there is one revealing piece of evidence that suggests the Earth received its water from somewhere quite cold. This evidence lies in the fraction of deuterium in water. Earth's water has an excess of deuterium (i.e., heavy water), and simple chemical principles inform us that this excess could only have been created when water is formed at a temperature of 10-20 K. In our Solar System, comets and asteroids all carry this signature and, in principle, we can then use the deuterium 
fraction to trace back the primary source of Earth's water to either the asteroid belt or larger distances where comets reside. Unfortunately, we currently have measurements toward only a handful of comets, all hinting at subtle $\mathrm{D} / \mathrm{H}$ variations. By making measurements toward hundreds of comets, Origins will finally establish whether asteroids and/or comets were the source of Earth's water. The third science objective in this key mission design science program will target close to 100 comets during the 5-year lifetime to measure $\mathrm{D} / \mathrm{H}$ (Table 1). The combination of these three objectives and their proposed measurements focused primarily on water and HD1-0 will transform our understanding of Earth's evolution as well as the mechanism by which habitable planets form and obtain the key life-enabling ingredient, water.

\subsection{Do planets orbiting M-dwarf stars support life?}

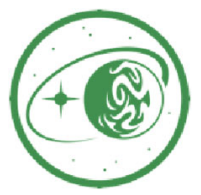

Are we alone? is one of the most compelling questions humankind has asked itself for millennia. The first exoplanets were detected only a few decades ago and since then, we have discovered thousands more. Some of these exoplanets are even in the habitable zone, where planetary surface temperatures are suitable for liquid water. But the question of whether they harbor life remains unanswered. The most promising approach is to search for biosignatures - pairs of molecules found in concentrations that are inconsistent with chemical equilibrium predictions. These molecules are presumed to be replenished by some form of life. Only recently have we been able to build instruments sensitive and stable enough to search for biosignatures in an exoplanet's atmosphere. Origins is equipped with a dedicated mid-infrared instrument for emission and transmission spectroscopy over a large wavelength range and achieving unprecedented stability. Thus, Origins will be able to expand upon the legacies of Hubble and Spitzer - and soon JWST - by searching for biosignatures in the atmospheres of transiting exoplanets orbiting M dwarfs (Fig. 5), the most abundant stars in the Galaxy. Origins follows a three-tiered approach, starting with a sample of exoplanets with welldetermined masses and radii, and narrowing that sample down to candidates where the detection of atmospheric biosignatures is most likely.

What fraction of terrestrial K- and M-dwarf planets has tenuous, clear, or cloudy atmospheres? In the first step, Origins will measure transmission spectra over $2.8 \mu \mathrm{m}-20 \mu \mathrm{m}$ to establish the fraction of temperate terrestrial exoplanets with clear, cloudy, and tenuous atmospheres. The sample will span a large range of planet sizes, equilibrium temperatures, and orbital distances, and can include planets orbiting stars from late-K to late-M type, giving Origins a broader perspective in the search for life than JWST.

What fraction of terrestrial M-dwarf planets is temperate? For the subset of planets with clear atmospheres, Origins will measure their emission spectra to determine the temperature structure of the atmosphere and hence the absorption and emission of stellar radiation. The temperature profiles can then identify planets suitable to contain liquid water on their surfaces [12]. 


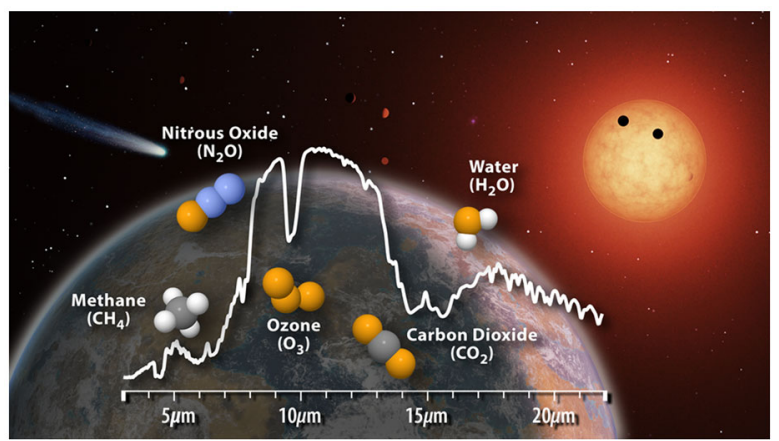

Fig. 5 Origins is designed to search for atmospheric biosignatures of exoplanets that transit K- and M-dwarf stars. By leveraging the mid-infrared wavelength capabilities with a dedicated state-of-the-art instrument for exoplanet transit and eclipse studies, Origins will study the exoplanet atmospheres for gases that are the most important signatures of life. (Figure reproduced from Fig. 6 in Meixner et al. [15]

What types of temperate, terrestrial M-dwarf planets support life? Origins will be the first observatory that cannot only measure the habitability indicators $\left(\mathrm{H}_{2} \mathrm{O}\right.$ and $\left.\mathrm{CO}_{2}\right)$, but also the biosignatures $\left(\mathrm{O}_{3}\right.$ coupled with $\mathrm{N}_{2} \mathrm{O}$ or $\left.\mathrm{CH}_{4}\right)$, which are the fingerprints of life. Origins will search for these biosignatures in the atmospheres of the most promising temperate planets. Due to its wide wavelength coverage, Origins can measure several transitions of each molecule, thus granting unambiguous identification. With its sensitive and stable mid-infrared spectrometer, Origins can investigate a variety of potentially habitable atmospheres, from the life-bearing Archaean Earth to today's modern Earth. For the first time, we will be able to probe the atmospheres of planets of all ages and to discover Nature's creativity to form life under different conditions elsewhere in our Galaxy [8].

\subsection{Discovery science for origins in its baseline configuration}

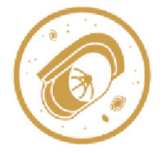

With more than three orders of magnitude improvement in sensitivity over Herschel and access to a spectral range spanning nearly 8 octaves, Origins vastly expands discovery space available to the community. While the mission is designed to achieve a specific set of objectives, the science program is intended to be illustrative only. Origins is a community observatory, driven by science proposals selected through the usual peer-review process, as with existing NASA observatories.

Suggestions for the discovery science for Origins include:

- Detection of warm molecular hydrogen from reionization

- Mapping Galaxy outflows in the nearby Universe

- Wide-field mapping of molecular hydrogen in local group dwarf galaxies

- Mapping magnetic fields at galactic scales

- Follow-up and characterization of LISA and LIGO gravitational-wave sources and other time-domain sciences 
- Time-domain sciences: proto-star variability as a probe of protoplanetary disc physics and stellar assembly

- Small bodies in the trans-Neptunian region: constraints on early Solar System cometary source region evolution

- Origins studies of water ice in non-disc sources

- Studying magnetized, turbulent, molecular clouds

- Below the surface: A deep dive into the environment and kinematics of low luminosity protostars

- Putting the Solar System in context: the frequency of true Kuiper-belt analogues

- Giant planet atmospheres: templates for brown dwarfs and exoplanets

Origins' sensitivity exceeds that of its predecessor missions by a factor of 1000. Jumps of this magnitude are very rare in astronomy, and have always revolutionized our understanding of the Universe in unforeseen ways. Thus, it is essentially guaranteed that the most transformative discoveries of Origins are not even anticipated today.

\subsection{Discovery science requiring HERO}

The Origins baseline concept proposes three extremely powerful instruments: OSS, FIP, and MISC. However, these instruments are incapable of fully investigating the trail of water from the cold interstellar medium to planet forming discs and Solar System objects.

\subsubsection{Early stages of the trail of water}

With its heterodyne receiver, Origins in its upscope configuration will play a critical role in tracing the early path of water from the ISM into young circumstellar discs through its unique access to the lowest-energy rotational transitions of the water molecule and its isotopologues $\left(\mathrm{H}_{2}{ }^{18} \mathrm{O}, \mathrm{H}_{2}{ }^{17} \mathrm{O}\right.$, HDO) at high spectral resolving power (up to $10^{7}$ ), and in synergy with JWST for tracing water ice through its infrared and far infrared bands. With the high sensitivity provided by its large, 5.9-m telescope and HERO's extremely high spectral resolution capabilities, Origins will be a transformational tool for following the path of water in the ISM.

While some interstellar water is known to be present in diffuse molecular gas and UV-irradiated photodissociation regions, the bulk of water is found in dense molecular clouds as ice mantles on cold $(\mathrm{T} \sim 10 \mathrm{~K})$ dust grains with tiny traces of water vapor (three orders of magnitude less abundant than water ice). Via a host of observations (e.g., $[2,17,25]$, it is now known that the water ice mantle first forms in pre-stellar cores. This is the water that is provided to the young disc and sets the stage for all that follows.

State-of-the-art chemo-dynamical models of the prestellar core evolution that include water ice and cosmic ray-induced production of water vapor predict that, overall, for a typical prestellar core of 1 solar mass $\left(2 \times 10^{33} \mathrm{~g}\right)$, the total mass of water vapor can be estimated to lie between 20 and $2 \times 10^{3}$ Earth ocean mass, while the total mass including water ice would be up to a few millions Earth ocean mass. The spread in these estimates is not only due to the individual variation between cores related to their environment, but also to the lack of adequate observing facilities since Herschel. 
There is only a single published observation of detection of water vapor in a starless core [3] that highlighted the role of cosmic rays and provided definitive evidence for a gravitationally collapsing coresee Fig. 6. Because of their sensitivity to hydrogen densities higher than $10^{7} \mathrm{~cm}^{-3}$, the ground state water line profiles uniquely reveal the dynamics of the inner regions of the collapsing core, enabling a clear and accurate test of star formation theories and an accurate measurement of the amount, radial distribution and infall speed of water that is delivered to the forming protostar/ protoplanetary system $[9,10]$. The main gas phase precursors, $\mathrm{OH}$ and $\mathrm{H}_{3} \mathrm{O}^{+}$, will be accessible to HERO, leading to a complete account of the chemical network of water. This will allow us to follow the formation of water during the dynamical evolution of starless cores, on their way of star and stellar system formation. Measurements of water vapor in different environments will also provide important clues on how physical parameters (e.g. the impinging radiation field, volume densities, dust and gas temperatures and turbulent content) affect the production of water and its accumulation on dust grains, the building blocks of pebbles and planets. This is crucial to put stringent constraints on our chemical-dynamical models. With its upgraded instrument suite Origins is uniquely suited to perform a deep survey of cores at different stages of evolution and in different star-forming environments.

HERO is a powerful instrument to zoom into the disc using line-tomography:

- The high spectral resolving power of $30 \mathrm{~m} / \mathrm{s}$ allows much more detailed localization of the gas than OSS, especially for the cold regions in large discs, which have Keplerian velocities as low as a $1-2 \mathrm{~km} / \mathrm{s}$.

- Line tomography will be possible over the whole frequency range of HERO, including the lowest-lying ortho-water line at $538 \mu \mathrm{m}$, which traces the coldest

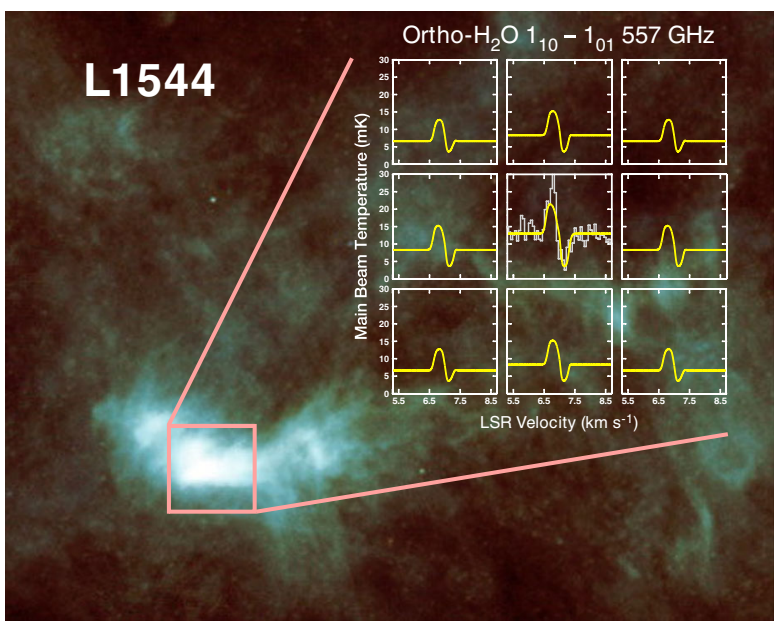

Fig. 6 High spectral resolution observations reveal complex line shapes of the ground state water line that allow us to determine the dynamics of the inner cores. The L1544 prestellar core showing the dust continuum emission. The insert is the modeled line profiles of the $\mathrm{H}_{2} \mathrm{O} 1_{10}-1_{01}$ transition at $538 \mu \mathrm{m}(557 \mathrm{GHz})$ in the $25^{\prime \prime}$ FWHM beam of Origins using the MOLLIE radiative transfer code [9]. Each panel is separated by 25". The central panel includes the HIFI spectrum with a 40" beam. Note the higher continuum level and deeper absorption in the central panel, which shows the forming core 
gas $(\mathrm{E} / \mathrm{k}=27 \mathrm{~K})$, and is significantly less effected by dust attenuation than the 179.5 micron line, giving access to deeper layers, even in massive discs.

- A single setting of HERO observes both the ortho-line at $538 \mu \mathrm{m}$, and the para-line at $269 \mu \mathrm{m}$, and includes the entire line profile with at least 100 resolution elements without the need for scanning.

\subsubsection{Final stages of the trail of water}

HERO provides high spectral resolution observations of water in our Solar System that allows us to understand in detail how water was transported to Earth and where it is found in our Solar System. We can apply this knowledge to other planetary systems also to help our search for life.

Comets are one possible origin of water on Earth, where the $\mathrm{D} / \mathrm{H}$ ratio is an important indicator of the likelihood that comets delivered water found on Earth [5]. Complementary to the large OSS comet survey, HERO carries out follow up observations of the brighter comets at very high spectral resolving power $\left(10^{7}\right)$ in order to determine the origin of outgassing for different molecules (by analyzing the line profiles), to get a refined $\mathrm{D} / \mathrm{H}$ ratio, to determine the excitation mechanisms of the gases, and to determine the gas coma structure. HERO contributes to questions about the origin of the Solar System by providing isotopic ratios (e.g. $\mathrm{D} / \mathrm{H},{ }^{16} \mathrm{O} /{ }^{17} \mathrm{O}$ and ${ }^{16} \mathrm{O} /$ ${ }^{18} \mathrm{O}$ ) for a large number of comets and linking the age of these objects to other primitive bodies.

Water does not only exist on Earth, but also on other planets, such as Mars, which may have supported life. HERO observations constrain the water cycle [22], hydrogen/ oxygen chemistry and origin of the Martian atmosphere by very sensitive and highly spectrally-resolved observations of molecules and their isotopologues [24] and provide their vertical profiles from the pressure broadened line shapes. Furthermore it will provide upper limits for a large number of molecules so far not detected (e.g. $\mathrm{HCl}$ ). HERO constrains the origin of water in the stratosphere of the gas and ice giants, determines the $\mathrm{D} / \mathrm{H}$ ratio in hydrogen and water and the isotopic ratios of at least $\mathrm{C}, \mathrm{S}$ and $\mathrm{O}$ with high precision. Moons of these planets also contain water. HERO is capable of monitoring the composition, physical conditions and variability of the Enceladus torus [6], the water atmospheres of the Galilean satellites (including detection of plumes), Titan [16] and the dwarf-planet Ceres [11].

\subsubsection{Origins as an event horizon telescope station}

The concept of using Origins to study black hole physics on event horizon scales was recently raised as a potential extension of the HERO science case [18] that shows much promise and merits further study. The unprecedented angular resolution resulting from the combination of Origins with existing ground-based submillimeter/millimeter telescope arrays would increase the number of spatially resolvable black holes by a factor of $10^{6}$, permit the study of these black holes across all cosmic history, and enable new tests of General Relativity by unveiling the photon ring substructure in the nearest black holes, see Fig. 7. Expanding the HERO instrument to be an interferometric station will 

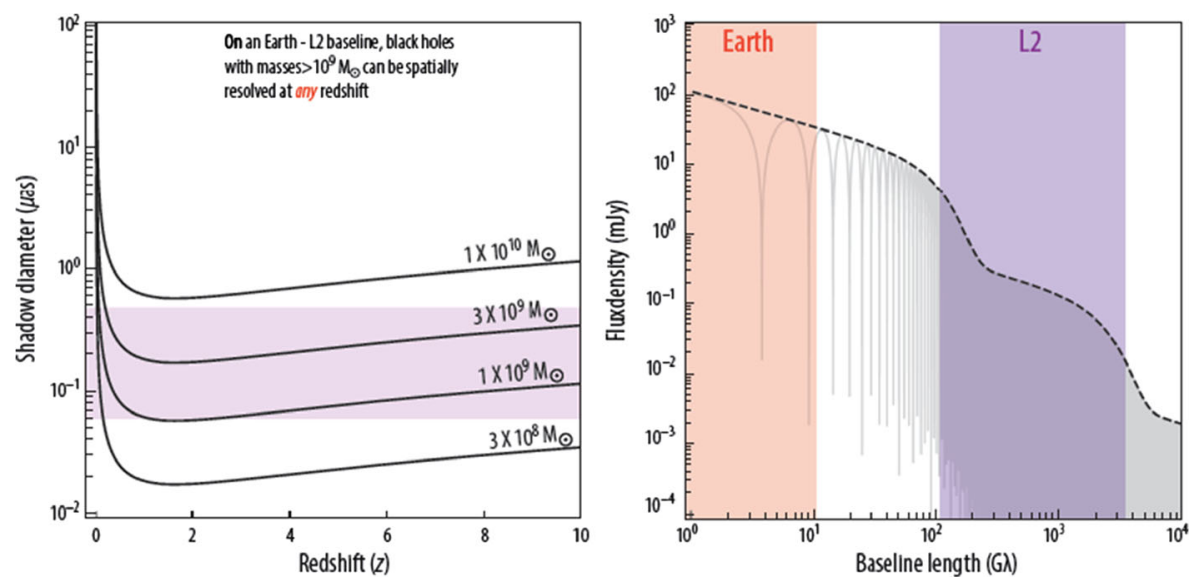

Fig. 7 Left: Black hole shadow diameter versus redshift for SMBHs. Right: Flux density as a function of interferometer baseline length. In both plots the parameters for the L2-Earth baseline is shaded in purple

require small technology enhancements. (For more details: Origins Space Telescope Mission Concept Study Report, App D-16.)

\subsection{Discovery science requiring MISC-upscope}

\subsubsection{The rise of metals}

Origins/OSS will use sensitive metallicity indicators in the infrared to track the growth history of dust. At $\mathrm{z}>5$ galaxies are generally metal poor and bright rest-frame optical lines as well as the $3.3 \mu \mathrm{m}$ PAH feature that fall into the mid-IR are best suited to trace the dust. The $3.3 \mu \mathrm{m}$ PAH feature is particularly interesting as it can be used to track metallicity as long as it is not destroyed by high UV radiation fields typically found in low metallicity environments. The MISC upscope spectrometer allows these observations. Observations of different mid-IR emission lines also carry information about the nature of photo-ionization in individual galaxies.

\subsubsection{Building the stellar masses of early galaxies (Roman + Origins/MISC)}

Stars emit photons over the entire wavelength range, but the unreddened emission from stars in the early Universe at $5<\mathrm{z}<10$ falls in the rest-frame UV, optical and near-IR (roughly $0.25-3.5 \mu \mathrm{m}$ ). This information is used to estimate the star formation rate (SFR). To get a picture of the history of stellar formation, we also need to measure the integral of this SFR (t), e.g., the stellar mass of galaxies. This translates into wavelengths that uniquely match Origins/MISC's, i.e. 5 - $30 \mu \mathrm{m}$. The rest-frame UV spectrum will provide an access to young stars that are likely to be predominant at $z>5$. We can collect this information from Roman-Deep and Roman-Wide surveys. But, if we want to perform a complete census, included potential older stars, we need the rest-frame optical and near-IR. To follow up Roman's objects at $z>5$, after JWST's lifetime, we need an instrument like MISC on Origins. The ELT might bring some information, though, but mainly below $2.5 \mu \mathrm{m}$. Using Origins/MISC to study the 
galaxies detected at $5<\mathrm{z}<10$ in Roman-Deep and Roman-Wide surveys will enable critical measurements of the star formation rate (SFR), stellar mass (M), and dust attenuation. Two surveys using Origins/MISC WFI photometric and spectroscopic capabilities will provide unique data that broaden our understanding of the evolution of the mass function (cosmic mass assembly), star formation rate density, and average dust attenuation for a representative sample of galaxies at $5<\mathrm{z}<10$.

The WFI-L channel $(9-28 \mu \mathrm{m})$ is prioritized rather than WFI-S $(5-9 \mu \mathrm{m})$ in the course of the descope discussion by the Science and Technology Definitions Team (STDT).

\subsection{Origins: a mission for the astronomical community}

Unanticipated, yet transformative, discovery space: The impressive Origins-enabled scientific advances discussed above are extensions of known phenomena. However, history has shown that order-of-magnitude leaps in sensitivity (Figs. 8 and 9) lead to discoveries of unanticipated phenomena. For example, the sensitivity of IRAS over balloon and airborne infrared telescopes allowed the discovery of debris discs, protostars embedded within dark globules, Galactic infrared cirrus, and IR-bright galaxies, none of which were expected at the time of launch. Likewise, no study anticipated that Spitzer would study $z>6$ galaxies, measure winds transporting energy in exoplanet atmospheres, and detect the dust around white dwarfs produced by shredded asteroids.

\section{Technical overview}

\subsection{Science traceability}

The three main science themes define the science traceability matrix (Table 2) for the Origins Space Telescope design.

Origins is $>1000$ times more sensitive than prior far-infrared missions and the design avoids complicated deployments to reduce mission risk. The scientific objectives summarized in Table 1 are achievable with the low-risk Origins design. Origins has a Spitzerlike architecture (Fig. 10) and requires only a few simple deployments to transform from launch to operational configuration. With the attributes shown in Table 3, the current design carries significant margin between science-driven measurement requirements and estimated performance (Table 2), leaving room for modest descopes.

Origins provides a thousand-fold improvement in the far-infrared sensitivity relative to Herschel (Figs. 8 and 9). While Origins has $2.8 \times$ Herschel's collecting area, cryocooling is the dominant factor enabling its extraordinary sensitivity gain. To achieve the same sensitivity gain at optical wavelengths, the light-collecting area would have to increase a thousand-fold. A far-IR telescope limited in sensitivity by the astronomical background is essential to achieving the Origins science goals. Earth's warm atmosphere limits SOFIA's sensitivity and Herschel was limited by a relativelywarm telescope $(70 \mathrm{~K})$. The cryo-thermal system design of Origins leverages Spitzer experience and technology developed for JWST. Four current-state-of-the-art cryocoolers cool the telescope to $4.5 \mathrm{~K}$, with $100 \%$ margin in heat-lift capacity at each stage. The science requirements can be met with a telescope temperature below $6 \mathrm{~K}$. 
The telescope is diffraction limited at $30 \mu \mathrm{m}$. All of the telescope's mirrors and mirror segments can be diamond turned and rough polished to the required precision in existing facilities. The JWST primary mirror segment actuator design is reused, to allow the Origins primary mirror segments to be adjusted in space in three degrees of freedom (tip, tilt, and piston), enabling final alignment during commissioning. The telescope is used as a light bucket at wavelengths between $2.8 \mu \mathrm{m}$ and $20 \mu \mathrm{m}$ to perform transit spectroscopy for exoplanet biosignatures since spatial resolution is not a technical driver for that scientific objective.

The Origins design minimizes complexity. The optical system launches in its operational configuration, requiring no mirror, barrel, or baffle deployments after launch, but the design allows for mirror segment alignment on orbit to optimize performance. The two-layer sunshield deployment is simple and low risk and other deployment mechanisms - communication antenna, solar array, telescope coverhave extensive heritage. This departure from the JWST deployment approach is enabled by the capabilities of new launch vehicles, which are expected to be fully operational in the mid-2030s. The design is compatible with at least two, and possibly three such launch vehicles. The fully-integrated cryogenic payload assembly comprising the telescope, instruments, and cold shield can be tested cryogenically in Chamber A at NASA's Johnson Space Center, following NASA's favored "test-like-you-fly" approach.

The next generation of launch vehicles, including NASA's SLS, SpaceX's BFR, and Blue Origin's 7-m New Glenn, have much larger payload fairings than the 5-m diameter ones available today, enabling the launch of a large-diameter telescope that does not need to be folded and deployed. Origins operates in a quasi-halo orbit around the Sun-Earth L2 point. The observatory is robotically serviceable, enabling future instrument upgrades and propellant replenishment to extend the mission life beyond the 5-year design lifetime.
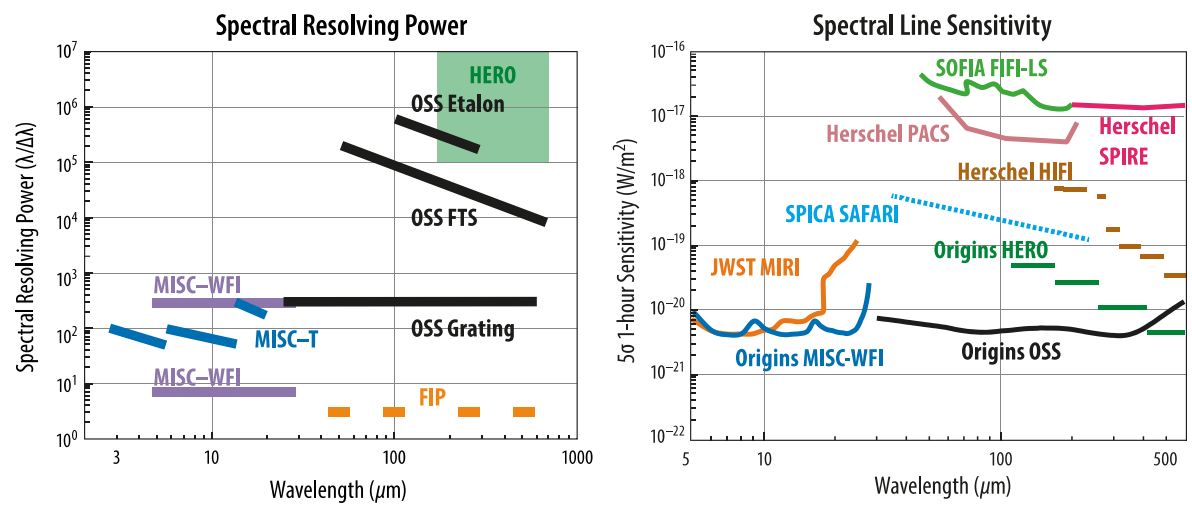

Fig. 8 Origins has a comprehensive set of three baseline instruments and two upscope instrument options that make it a very versatile and powerful instrument for key science questions as well as unanticipated discoveries. Origins surpasses all prior, current, and planned missions by a large factor setting the stage for a new era of far-IR astronomy. (Figure reproduced from Fig. 13 in Leisawitz et al. [14] 


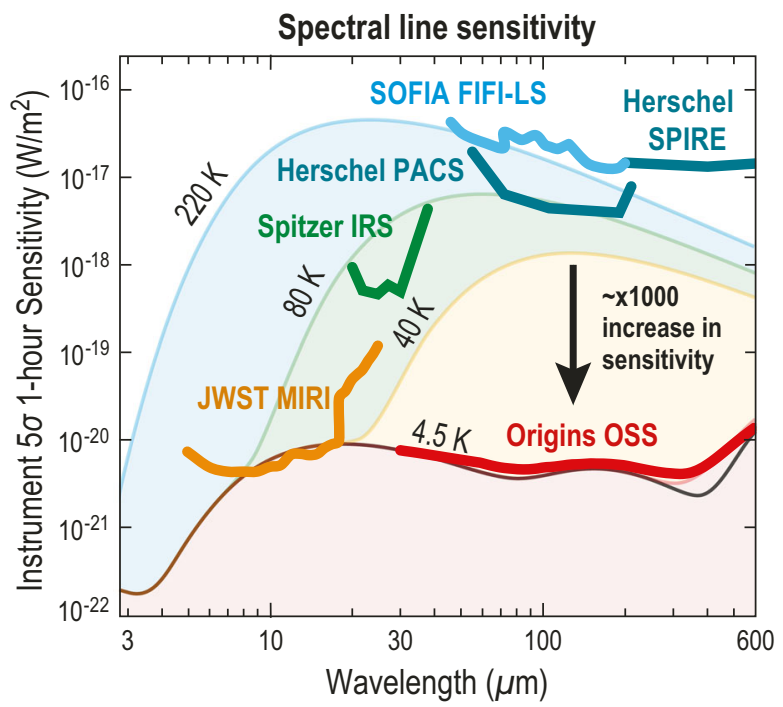

Fig. 9 Origins taps into a vast, unexplored scientific discovery space, defined by a three-orders-of-magnitude improvement in sensitivity relative to all previously-flown far-infrared observatories. With a temperature of 4.5 K, Origins' sensitivity is limited by astronomical background photon noise (lower black curve). SOFIA $(220 \mathrm{~K})$, Herschel $(80 \mathrm{~K})$, and JWST $(40 \mathrm{~K})$ are shown for comparison with Origins $(4.5 \mathrm{~K})$. Origins' sensitivity extends JWST/MIRI sensitivity in mid-IR to the far-IR wavelengths. (Figure reproduced from Fig. 12 in Leisawitz et al. [14]

\subsection{Origins instruments}

Three baseline science instruments spanning the wavelength range $2.8 \mu \mathrm{m}$ to $588 \mu \mathrm{m}$ provide the powerful, new spectroscopic and imaging capabilities required to achieve the scientific objectives, see Table 4.

OSS is a highly capable spectrometer that covers the entire $25 \mu \mathrm{m}$ to $588 \mu \mathrm{m}$ band at moderate $(\mathrm{R} \sim 300)$, high $\left(\mathrm{R} \sim 4 \times 10^{4}\right)$, and ultra-high $\left(\mathrm{R} \sim 2 \times 10^{5}\right)$ spectral resolving power. OSS uses six gratings in parallel to take multi-beam spectra simultaneously across the $25 \mu \mathrm{m}$ to $588 \mu \mathrm{m}$ window through long slits. In this grating mode, OSS spatially and spectrally maps up to tens of square degrees of the sky providing 3-D data cubes. When needed, a Fourier transform interferometer and an etalon provide high and ultra-high spectral resolving power, respectively, in a single beam, with insertable elements that redirect the light path. The three OSS spectroscopy modes are packaged into one instrument. To meet its performance requires improved detector sensitivity and larger pixel format size.

FIP is a simple and robust instrument that provides imaging and polarimetric measurement capabilities at $50 \mu \mathrm{m}$ and $250 \mu \mathrm{m}$. FIP utilizes Origins' fast mapping speed (up to 60" per second) to map one to thousands of square degrees. FIP's images will be useful for telescope alignment and public relations. FIP's rapid mapping makes photometric variability studies possible for the first time. To meet its performance requires improved detector sensitivity and larger pixel format size. Three baseline science instruments spanning the wavelength range $2.8 \mu \mathrm{m}$ to $588 \mu \mathrm{m}$ provide 


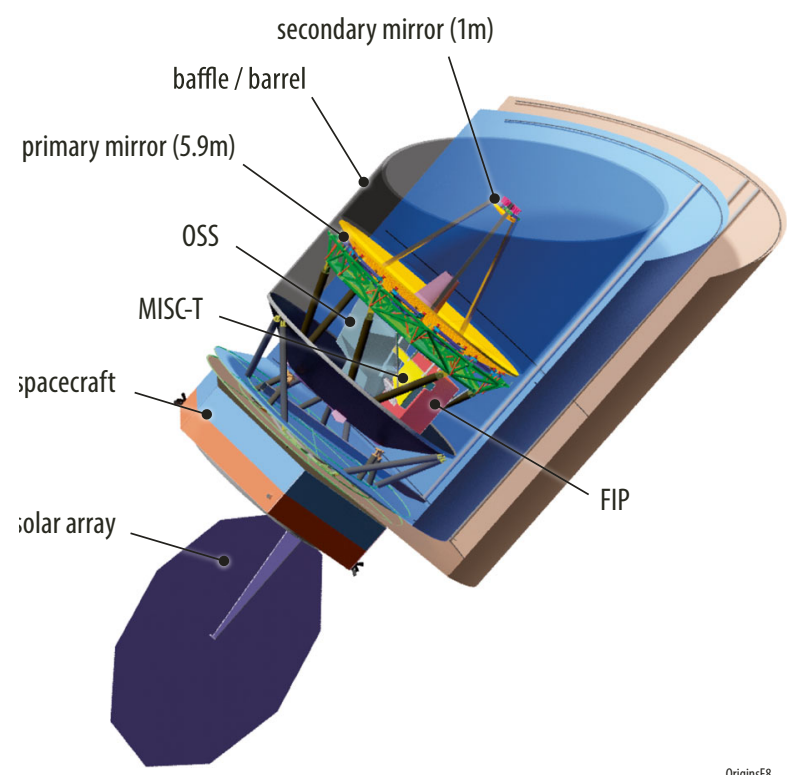

Fig. 10 Origins builds on substantial heritage from Spitzer to minimize schedule risks during assembly, integration and testing, and deployment risks in space. A cutaway view shows the locations of Origins instruments and major elements of the flight system. Origins, with an aperture diameter of $5.9 \mathrm{~m}$ and a suite of powerful instruments, operates with spectral resolving power from 3 to $3 \times 10^{5}$ over the wavelength range from $2.8 \mu \mathrm{m}$ to $588 \mu \mathrm{m}$. Origins has the agility to survey wide areas, the pointing stability required to observe transiting exoplanets, and operates with $>80 \%$ observing efficiency, in line with the approximately $90 \%$ efficiency achieved with Herschel and Spitzer. (Figure reproduced from Fig. 6 in Leisawitz et al. [14]

MISC-T measures $\mathrm{R} \sim 50$ to 300 spectra in the $2.8 \mu \mathrm{m}$ to $20 \mu \mathrm{m}$ band with three subsystems that operate simultaneously. MISC-T has no moving parts and is designed to provide exquisite stability and precision $(<5 \mathrm{ppm}$ between $2.8 \mu \mathrm{m}$ to $10 \mu \mathrm{m},<20 \mathrm{ppm}$

Table 3 Origins observatory-level parameters

\begin{tabular}{|l|l|}
\hline Mission Parameter & Value \\
\hline Telescope: Aperture Diameter/Area & $5.9 \mathrm{~m} / 25 \mathrm{~m}^{2}$ \\
\hline Telescope Temperature & $4.5 \mathrm{~K}$ \\
\hline Wavelength Coverage & $2.8 \mu \mathrm{m}-588 \mu \mathrm{m}$ \\
\hline Maximum Scanning Speed & $60^{\prime \prime}$ per second \\
\hline Mass: Dry/Wet (with margin) & $12000 \mathrm{~kg} / 13000 \mathrm{~kg}$ \\
\hline Power (with margin) & $4800 \mathrm{~W}$ \\
\hline Launch Year & 2035 \\
\hline Launch Vehicle & SLS Block IB or Space-X BFR \\
\hline Orbit & Sun-Earth L2 \\
\hline Propellant lifetime & $\begin{array}{l}10 \text { years, serviceable, limited by station-keeping } \\
\text { propellant }\end{array}$ \\
\hline
\end{tabular}


Table 4 Instrument capabilities summary

\begin{tabular}{|c|c|c|c|c|c|}
\hline $\begin{array}{l}\text { Instrument/ } \\
\text { Observing Mode }\end{array}$ & $\begin{array}{l}\text { Wavelength } \\
\text { Coverage }(\mu \mathrm{m})\end{array}$ & Field of View & $\begin{array}{l}\text { Spectral } \\
\text { Resolving } \\
\text { Power } \\
(R=\lambda / \Delta \lambda)\end{array}$ & $\begin{array}{l}\text { Saturation } \\
\text { Limits }\end{array}$ & $\begin{array}{l}\text { Representative sensitivity } \\
5 \sigma \text { in } 1 \mathrm{hr}\end{array}$ \\
\hline \multicolumn{6}{|c|}{ Baseline Instruments } \\
\hline \multicolumn{6}{|c|}{ Origins Survey Spectrometer (OSS) } \\
\hline Grating & $\begin{array}{l}6 \text { bands cover } \\
25-588 \mu \mathrm{m} \\
\text { simultaneously }\end{array}$ & $\begin{array}{l}6 \text { Slits: } \\
2.7^{\prime} \times 1.4^{\prime \prime} \text { to } \\
14^{\prime} \times 20^{\prime \prime}\end{array}$ & 300 & 5 Jy@ $128 \mu \mathrm{m}$ & $\begin{array}{l}3.7 \times 10^{-21} \mathrm{~W} \mathrm{~m}^{-2} \\
\text { @ } 200 \mu \mathrm{m}\end{array}$ \\
\hline $\begin{array}{l}\text { High Resolution } \\
\text { w/Fourier } \\
\text { Transform } \\
\text { Spectrometer }\end{array}$ & $\begin{array}{l}25-588 \mu \mathrm{m} \\
\text { Total range } \\
\text { scanned by FTS }\end{array}$ & $\begin{array}{l}\text { Slit: } 20^{\prime \prime} \times 2.7 \text { to } \\
20^{\prime \prime} \times 20^{\prime \prime}\end{array}$ & $\begin{array}{l}43,000 \times \\
{[112 \mu \mathrm{m} / \lambda]} \\
\text { tunable } \\
\text { w/FTS scan } \\
\text { length }\end{array}$ & 5 Jy @ $128 \mu \mathrm{m}$ & $\begin{array}{l}7.4 \times 10^{-21} \mathrm{~W} \mathrm{~m}^{-2} \\
\text { @ } 200 \mu \mathrm{m}\end{array}$ \\
\hline $\begin{array}{l}\text { Ultra-High- } \\
\text { resolution } \\
\text { w/Fabry-Perot } \\
\end{array}$ & $\begin{array}{l}100-200 \mu \mathrm{m} \\
\text { Select lines } \\
\text { scanned }\end{array}$ & One beam: 6.7" & $\begin{array}{l}325,000 \times \\
{[112 \mu \mathrm{m} / \lambda]}\end{array}$ & $\begin{array}{l}100 \text { Jy @ } 180 \\
\mu \mathrm{m}\end{array}$ & $\begin{array}{l}\sim 2.8 \times 10^{-19} \mathrm{~W} \mathrm{~m}^{-2} \\
@ 200 \mu \mathrm{m}\end{array}$ \\
\hline \multicolumn{6}{|c|}{ Far-infrared Imager Polarimeter (FIP) } \\
\hline Pointed & $\begin{array}{l}50 \text { or } 250 \mu \mathrm{m} \\
\text { (selectable) }\end{array}$ & $\begin{array}{l}50 \mu \mathrm{m}: 3.6^{\prime} \times 2.5^{\prime} \\
250 \mu \mathrm{m}: 13.5^{\prime} \times 9^{\prime} \\
(109 \times 73 \text { pixels })\end{array}$ & 3.3 & $\begin{array}{l}50 \mu \mathrm{m}: 1 \mathrm{Jy} \\
250 \mu \mathrm{m}: 5 \mathrm{Jy}\end{array}$ & $\begin{array}{l}50 / 250 \mu \mathrm{m}: 0.9 / 2.5 \mu \mathrm{Jy} \\
\text { confusion limits } \\
50 / 250 \mu \mathrm{m}: 120 \mathrm{nJy} / \\
1.1 \mathrm{mJy}\end{array}$ \\
\hline Survey mapping & $\begin{array}{l}50 \text { or } 250 \mu \mathrm{m} \\
\text { (selectable) }\end{array}$ & $\begin{array}{l}60^{\prime \prime} \text { per second } \\
\text { scan rate, with } \\
\text { above FOVs }\end{array}$ & 3.3 & $\begin{array}{l}50 \mu \mathrm{m}: 1 \mathrm{Jy} \\
250 \mu \mathrm{m}: 5 \mathrm{Jy}\end{array}$ & $\begin{array}{l}\text { Same as above, time to } \\
\text { reach confusion limit: } \\
50 \mu \mathrm{m}: 1.9 \text { hours } \\
250 \mu \mathrm{m}: 2 \text { millisec }\end{array}$ \\
\hline Polarimetry & $\begin{array}{l}50 \text { or } 250 \mu \mathrm{m} \\
\text { (selectable) }\end{array}$ & $\begin{array}{l}50 \mu \mathrm{m}: 3.6^{\prime} \times 2.5^{\prime} \\
250 \mu \mathrm{m}: 13.5^{\prime} \times 9^{\prime}\end{array}$ & 3.3 & $\begin{array}{l}50 \mu \mathrm{m}: 10 \mathrm{Jy} \\
250 \mu \mathrm{m}: 10 \mathrm{Jy}\end{array}$ & $\begin{array}{l}0.1 \% \text { in linear polarization, } \\
\pm 1^{\circ} \text { in pol. Angle }\end{array}$ \\
\hline \multicolumn{6}{|c|}{ Mid-Infrared Spectrometer Camera Transit Spectrometer (MISC-T) } \\
\hline $\begin{array}{l}\text { Ultra-Stable } \\
\text { Spectroscopy }\end{array}$ & $\begin{array}{l}2.8-20 \mu \mathrm{m} \text { in } 3 \\
\text { simultaneous } \\
\text { bands }\end{array}$ & $\begin{array}{l}2.8-10.5 \mu \mathrm{m}: 2 . " 5 \\
\text { radius } \\
10.5-20 \mu \mathrm{m}: 1 . " 7 \\
\text { radius }\end{array}$ & $\begin{array}{l}2.8-10.5 \mu \mathrm{m}: \\
50-100 \\
10.5-20 \mu \mathrm{m}: \\
165-295\end{array}$ & 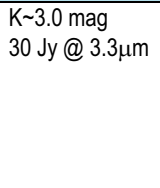 & $\begin{array}{l}\text { Assume } \mathrm{K} \sim 9.85 \text { mag } \mathrm{M} \text { - } \\
\text { type star, } \mathrm{R}=50 \\
\mathrm{SNR} / \mathrm{sqrt}(\mathrm{hr})>12,900 @ 3.3 \\
\mu \mathrm{m} \text { in } 60 \text { transits with } \\
\text { stability } \sim 5 \mathrm{ppm}<10.5 \mu \mathrm{m} \text {, } \\
\sim 20 \mathrm{ppm}>10.5 \mu \mathrm{m}\end{array}$ \\
\hline \multicolumn{6}{|c|}{ Upscope Instruments } \\
\hline \multicolumn{6}{|c|}{ HEterodyne Receiver for Origins (HERO) } \\
\hline $\begin{array}{l}\text { Spectral line } \\
\text { observations }\end{array}$ & $\begin{array}{l}110 \text { to } 620 \mu \mathrm{m}, \\
\text { dual-frequency, } \\
\text { dual-polarization }\end{array}$ & $0 .{ }^{\prime} 5 \times 0 . .^{\prime} 5$ to $2^{\prime \prime} \times 2^{\prime \prime}$ & up to $10^{7}$ & NA & $\begin{array}{l}6.4 \times 10^{-21} \text { at } 480 \mu \mathrm{m} \\
7.3 \times 10^{-20} \text { at } 130 \mu \mathrm{m}\end{array}$ \\
\hline \multicolumn{6}{|c|}{ Mid-Infrared Spectrometer Camera } \\
\hline Imaging & $5-28 \mu \mathrm{m}$ & $3^{\prime \prime} \times 3^{\prime \prime}$ & $5-10$ & TBD & $6.4 \times 10^{-21}$ at $480 \mu \mathrm{m}$ \\
\hline Spectroscopy & $5-28 \mu \mathrm{m}$ & $3^{\prime \prime} \times(0 . " 38$ to $1 . " 12)$ & 300 & 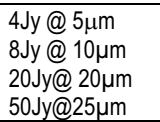 & $7.3 \times 10^{-20}$ at $130 \mu \mathrm{m}$ \\
\hline
\end{tabular}

$11 \mu \mathrm{m}$ to $20 \mu \mathrm{m})$. The optics design uses densified pupil optics that mitigate for observatory jitter. The improved stability relies on a planned improvement in detector stability including calibration. 
The Origins mission concept study team also developed two upscopes instruments, which enhance the mission's scientific capability: The Heterodyne Receiver for Origins (HERO) and the MISC Camera module. HERO provides nine-beam spectral measurements of selectable lines between $110 \mu \mathrm{m}$ and $620 \mu \mathrm{m}$ bands, up to very high spectral resolving power around $10^{7}$. The MISC Camera enables mid-infrared imaging and spectroscopy $(R=300)$ between $5 \mu \mathrm{m}$ and $28 \mu \mathrm{m}$. In addition there are upscopes for the existing instruments including expanded FOVs for OSS and FIP, and additional FIP bands $(100 \mu \mathrm{m}$ and $500 \mu \mathrm{m})$. Potential descopes include reducing the baseline instruments' modes and decreasing the aperture diameter, and would impact the observatory's science capabilities.

\section{Key technologies}

Detectors, ancillary detection system components and cryocoolers are the only Origins enabling technologies currently below Technology Readiness Level (TRL) 5. The Origins Technology Development Plan outlines a path leading to TRL 5 by Phase A start in 2025.

At far-infrared wavelengths, reaching the fundamental sensitivity limits set by the astronomical background (Fig. 8) requires a cold telescope equipped with sensitive detectors. The noise equivalent power (NEP) required for FIP imaging is $3 \times 10^{-}$ $19 \mathrm{~W} \mathrm{~Hz}^{-1 / 2}$, whereas the NEP needed for OSS for $R=300$ spectroscopy is $3 \times 10^{-}$ ${ }^{20} \mathrm{~W} \mathrm{~Hz}^{-1 / 2}$. Transition edge sensor (TES) bolometers and kinetic inductance detectors (KIDs) both show great promise, and the plan is to mature both technologies to TRL 5 and then down-select to a single technology at the beginning of Phase A. While the noise requirements for MISC-T's mid-IR detectors are not particularly challenging, 5 ppm stability over several hours must be demonstrated to meet the Origins requirement. The Technology Development Plan mitigates risk by recommending the parallel maturation of $\mathrm{HgCdTe}$ arrays, Si:As arrays, and TES bolometers (Fig. 11).

For the MISC upscope large format Si:As arrays and a deformable mirror capable of operation at $\sim 8 \mathrm{~K}$ are required. The increase in Si:As array format size is expected to be relatively straightforward as the JWST MIRI instrument already has detectors with the required performance, albeit a smaller array size. A cryogenic deformable mirror has been demonstrated, but at slightly higher temperatures.

The HERO upscope is based on the successful Herschel/HIFI receiver and is low risk. However, the HERO design uses the latest innovative components in order to substantially reduce weight, cooling power, and electrical power so that HERO can fly the first heterodyne array receivers on a satellite. Development of these components has already started, but needs to be continued to reach TRL 5 in 2025. The R\&D includes broadband (hot electron bolometers and Superconducting Insulating Superconducting) mixers near the quantum limit, wideband local oscillators, low power, low noise cryogenic amplifiers, low power spectrometers and broadband optics.

Mechanical cryocoolers that can reach temperatures of $4.5 \mathrm{~K}$ have already flown on Hitomi (2016). These coolers, developed by Sumitomo Heavy Industries, had a required lifetime of 5 years compared to Origins' 10 years, but meet its performance requirements. Replacing the compressors' suspension system with a flex spring, a 


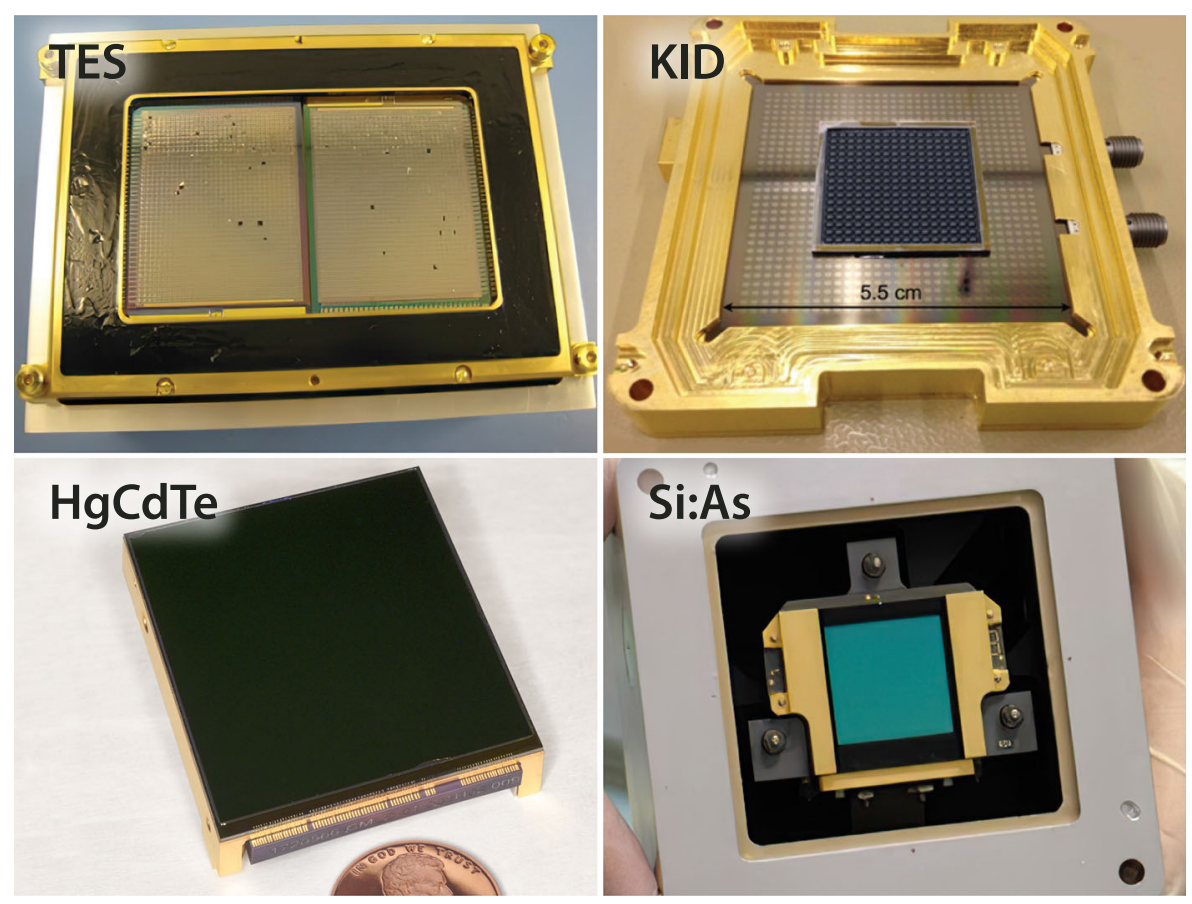

Fig. 11 The robust Origins Technology Development Plan recommends parallel maturation of multiple promising detector technologies (clock-wise from top-left): TES bolometers (HAWC + array), KIDS (432pixel device), HgCdTe arrays (JWST/NIRCam), and Si:As (JWST/MIRI)

relatively straightforward change, will extend the lifetime. Several US companies have also produced TRL-5 cryocoolers or cryocooler components with a projected 10 -year lifetime. The TRL 7 JWST/MIRI cryocooler, for example, has a $6 \mathrm{~K}$ operating temperature. Sub-Kelvin coolers operating at $50 \mathrm{mK}$, as needed for the OSS and FIP detectors, were also flown on Hitomi. A Continuous Adiabatic Demagnetization Refrigerator (CADR) with a much higher cooling power ( $6 \mu \mathrm{W}$ vs. $0.4 \mu \mathrm{W}$ for Hitomi), suitable for Origins, is currently being developed to TRL 6 under a Strategic Astrophysics Technology (SAT) grant. This new SAT CADR will also demonstrate selfshielding of magnetic fields to $1 \mu \mathrm{T}$, making it compatible with superconducting detectors that demand an ambient field of $<30 \mu \mathrm{T}$. A straightforward extension of this ADR technology allows operations at even lower temperatures $(35 \mathrm{mK})$, with similar cooling power. Lowering the operating temperature is a simple way to improve TES detector sensitivity, should that become necessary during mission formulation.

\section{Schedule and cost}

The Origins team developed a mission design concept, technical approach, technology maturation plan, risk management approach, budget, and a master schedule compatible with NASA guidelines for the Decadal Study and grounded in NASA and industry experience from previous successful large Class A missions. 
Origins is a NASA-led mission, managed by a NASA Center, and includes domestic and international partners. JAXA, and a CNES-led European consortium were active participants in the mission concept study, with each contributing an instrument design. US domestic participants included GSFC, Ames, MSFC, JPL, and the industry (Ball, Northrop, Lockheed, and Harris).

Figure 12 shows Origins Phase A through E schedule. Scheduled milestones and key decision points are consistent with formulation and development for Class A missions. The schedule supports an April 2035 launch, and includes 10 months of funded reserve. Much of the design and development work progresses through parallel efforts, with OSS as the critical path.

NASA Goddard Space Flight Center's Cost Estimating and Modeling Analysis (CEMA) office developed a cost estimate using the industry standard PRICE-H parametric cost-modeling tool. The CEMA cost estimate is based on a detailed master equipment list (MEL) and the Integrated Master Schedule (IMS) shown in Fig. 12 for the Origins baseline design. The MEL assigns an appropriate Technology Readiness Level (TRL) to each component. The CEMA cost model assumes that all components have matured to at least TRL 5 by the start of Phase A in 2025, and to at least TRL 6 by the mission preliminary design review (PDR). A separate Origins Space Telescope Technology Development Plan describes the maturation of all mission-enabling technologies on this timeline and reports the cost of technology maturation. The study team's mission cost estimate includes mission definition and development, the flight segment, the ground segment, and mission and science operations for 5 years. The launch cost $(\$ 500 \mathrm{M}$ for the SLS launch vehicle, as advised by NASA Headquarters) is also included. Working independently, Goddard's Resource Analysis Office (RAO) estimated the mission cost using a top-down parametric model. RAO and CEMA are firewalled from each other, but they both referred to the same MEL and mission schedule. The RAO and CEMA cost estimates agree to within $24 \%$.

Origins is a "large" ( $>$ \$1.5B) mission using the Decadal Survey's terminology. The NASA Headquarters-appointed Large Mission Concept Independent Assessment Team (LCIT) is tasked with validating the cost estimates supplied by each of the four large missions studied with NASA support. The study teams have decided to wait for feedback from the LCIT before publishing detailed cost information. The Origins Final Study Report will provide an LCIT-validated mission cost estimate.

The Origins mission design has not been optimized, and optimization may lead to cost savings. Optimization is planned as a Phase A activity. Japan and several ESA member nations have significant relevant expertise and have demonstrated interest in the Origins mission. Foreign contributions are expected to reduce NASA's share of

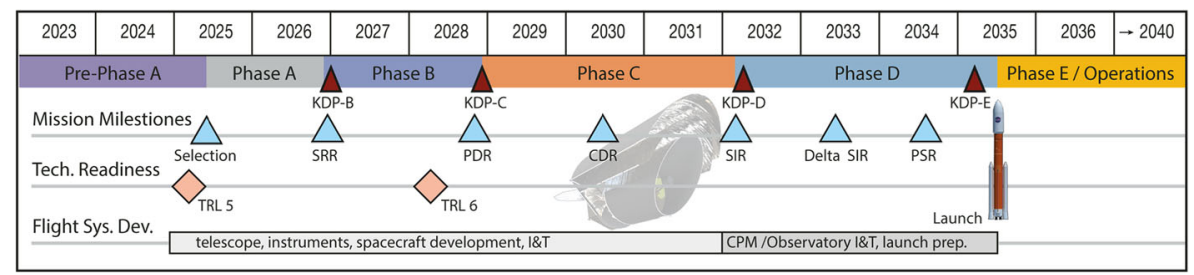

Fig. 12 Schedule for Origins Space Telescope 
the mission cost. NASA would welcome a European contribution equivalent in cost to an ESA M-class mission.

\section{Possible european contribution to origins}

Table 5 lists some possible European contributions. NASA has welcomed international and industry participation during the Origins study and has stated its interest in mission partnerships to be discussed in Phase A.

The segmented $5.9 \mathrm{~m}$ mirror could be contributed by Europe. Europe has extensive experience in fabricating Silicon Carbide mirrors for space. The $3.5 \mathrm{~m}$ mirror for Herschel and the $1.5 \times 0.5 \mathrm{~m}^{2}$ mirror for Gaia where fabricated in France and Germany.

The OSS and the FIPS instruments use detectors, readout and optics similar to that developed in Europe. A number of groups in the Netherlands and the United Kingdom are developing ultra-sensitive Kinetic Inductance Detectors (KID) and Transition-Edge Sensed (TES) bolometers, as well as the readout systems needed to operate these devices for SPICA/SAFARI and Athena/XIFU. The US design team has already contacted their European colleagues during the study to learn from each other. A potential partnership could be for one of the European groups to provide detector fabrication and delivery, after a common development period early in the project. A related possibility could be for the readout electronics, as French groups developed the readout for the Herschel/SPIRE and Planck/HFI bolometers, even though the detectors came from the US and were integrated into the instrument in the UK (SPIRE) and France (Planck/HFI).

Other possibilities include developing specific optical components. The etalon would be an excellent partnership opportunity since it is relatively modular and will require independent testing in advance of delivery. A group at SRON in the Netherlands is already developing new multi-layer mirrors that can make high-finesse etalons, and groups at Cardiff (UK) have experience with mirror materials and narrow-band

Table 5 Possible European Contribution

\begin{tabular}{|c|c|c|}
\hline & Possible Role & Description \\
\hline Mirror & lead & 5.9 m Silicon Carbide (SiC) mirror - Herschel heritage \\
\hline OSS/FIPS Detectors & collaboration & $\begin{array}{l}\text { Common development with US, European fabrication and } \\
\text { delivery, US integration and testing }\end{array}$ \\
\hline OSS/FIPS readout & lead & low power read-out electronics \\
\hline OSS optics & collaboration & $\begin{array}{l}\text { Etalon, FTS, grating, filters could be fabricated, tested and } \\
\text { delivered by Europe }\end{array}$ \\
\hline Sub-K cryocoolers & lead & $50 \mathrm{mK}$ cooler for OSS/FIPS \\
\hline HERO instrument & lead & $\begin{array}{l}\text { All heterodyne components are available in Europe though a } \\
\text { collaboration with the US is desirable }\end{array}$ \\
\hline MISC instrument & $\begin{array}{l}\text { collaboration with } \\
\text { Japan and US }\end{array}$ & $\begin{array}{l}\text { Components from Europe with JAXA taking the potential lead } \\
\text { role. }\end{array}$ \\
\hline FIP Instrument & collaboration or $\mathrm{PI}$ & far-IR detectors, cryogenic mechanisms \\
\hline OSS Instrument & collaboration & $\begin{array}{l}\text { Fourier-Transform Spectrometer scanning mechanism, the } \\
\text { etalon, far-IR detector arrays, quasi-optical components }\end{array}$ \\
\hline Concept Study & collaboration & \\
\hline Science & collaboration & \\
\hline $\begin{array}{l}\text { Mission Operation \& Data } \\
\text { Analysis }\end{array}$ & collaboration & \\
\hline Education/Outreach & collaboration & \\
\hline
\end{tabular}


etalon-based filters. These groups also have experience in Fourier Transform Spectrometers and are specialists in IR filters. They have delivered flight hardware, so would be well positioned to contribute to OSS.

French partners could provide the sub-K cryogenics. The $300 \mathrm{mK}$ systems for Herschel/ SPIRE and Herschel/PACS and the $100 \mathrm{mK}$ system for Planck came from France.

A largely European team designed the HERO instrument. Institutes in the Netherlands, Sweden, Germany, France, Italy and Spain are worldwide leaders in different components of heterodyne detector systems. They have contributed to the success of Herschel/HIFI as well as instruments on balloons, on SOFIA and for ground-based telescopes.

The MISC instrument was led by a Japanese consortium and NASA/ARC, but France has made science and technical contributions. A collaboration between Japan, the US, and Europe could enhance the instrument.

Suggested European R\&D in preparation for Origins:

In order to maximize the scientific outcome of the mission and to allow for a valuable European involvement, feasibility studies and technical R\&D in critical items should start as soon as possible.

As Europe (CNES) led the design of the Heterodyne REceiver for Origins (HERO), and as the European submillimeter community is very interested in spectroscopy, it offers itself as a potential European P.I. Instrument. The instrument is low risk as it has solid foundations thanks to the successful Herschel/HIFI instrument as well as smaller missions/projects. However, HERO would be the first heterodyne array receiver on a satellite and R\&D work is required to increase its TRL (technology readiness level). A detailed technology roadmap has been developed and written up for HERO that can be provided upon request. The most critical developments are:

- Mixers: increasing sensitivity, stability and bandwidth of Hot Electron Bolometers

- Local Oscillators: Increasing RF bandwidth of Schottky amplifier-multiplier chains that are used as local oscillators,

- Intermediate Frequency Chain: Develop low-noise cryogenic amplifiers that consume very little power $(<0.5 \mathrm{~mW}$ for $20 \mathrm{~dB}$ gain),

- Backends: Develop spectrometers that consume very little power $(<2 \mathrm{~W}$, for $8 \mathrm{GHz}$ bandwidth, 4 bits, 8 k channels),

- Optics: Broadband, low-loss optics, in particular lenslet arrays and dichroic filters.

Cryogenic amplifiers, backends and optics are not only essential for HERO, but also part of the other far-IR instruments, FIP, and OSS, on Origins.

The above-mentioned R\&D work would not only greatly benefit Origins, but also other far-IR missions, such as the far-IR single dish and space interferometers also submitted to the ESA Voyage 2050 call.

\section{Origins in a worldwide context of space and ground telescopes}

Origins builds on the heritage of Spitzer and Herschel, but surpasses them by a factor of 1000 in sensitivity in the mid to far-infrared wavelengths. Such a sensitivity gain allows an entirely new science discovery space with Origins that is impossible at UV/optical and X-ray wavelengths. Astronomers worldwide share the wish for a single dish mid/ 
far-IR mission and there are other proposed far-IR space missions: The Russian space agency proposes the Millimetron Space Observatory ${ }^{3}$ with a $10 \mathrm{~m}$ primary mirror cooled to about $35 \mathrm{~K}$. Millimetron is intended to harbor a comparable set of instruments, though these need to be contributed by Europe, Japan or the US. Should Millimetron launch as proposed before 2030 it could cover a large part of the Origins science. SPICA ${ }^{4}$ is another satellite similar to Origins, but, unfortunately, it is no longer a candidate for ESA's M5 mission. SPICA was to be a smaller $(2.5 \mathrm{~m}$ versus $5.9 \mathrm{~m}$ primary), slightly warmer ( $8 \mathrm{~K}$ versus $4.5 \mathrm{~K}$ ) and therefore a factor 100 less sensitive than Origins. Nevertheless, it could have done some preparatory science that Origins could have followed up with larger statistics. SPICA did not have a stable mid-IR spectrometer for the search for biosignatures, nor did it have measurement capability with very high spectral resolving power in the far-IR $\left(\mathrm{R} \sim 1000\right.$ versus $\mathrm{R} \sim 2 \times 10^{5} / 10^{7}$ with HERO upscope), so most of the trail of water and spectral line observations remained unique to Origins. JWST will cover the near to mid-IR, but not extend to the far-IR. Nevertheless, JWST will allow preparatory science, e.g. identification of exoplanets) and will complement Origins.

Other far-IR astronomy satellite proposals were submitted to the ESA Voyage 2050 call:

The "Far Infrared Spectroscopy Surveyor (FIRSS)" by Rigopoulou et al. [20], which is to carry out a survey of our Milky Way and some other galaxies in the main cooling lines of CI, CII, NI, and OI. It is a small $(1-2 \mathrm{~m})$ satellite with large $(4 \times 2 \times 64$ pixel $)$ heterodyne receiver arrays. It is complementary to Origins, as it is built to carry out large surveys. It proposes very similar heterodyne receivers as Origins and requires the same technology development roadmap.

"The Microwave spectro-polarimeter of Matter and Radiation Across Space and Time" proposed by Delabrouille et al. [4] will carry out a high-angular-resolution, highsensitivity spectro-polarimetric survey of the microwave sky between $0-2000 \mathrm{GHz}$ to observe the CMB as well as atoms and molecules over time in order to understand dark matter and dark energy better. The proposed satellite has quite a few similarities with Origins but observes at longer wavelengths.

The "TeraHertz Exploration and Zooming-in for Astrophysics (THEZA)" concept proposed by Gurvits et al. [7] focuses on the physics of spacetime in the vicinity of black holes but also aims at a wide range of science topics from cosmology to origins of life in the Universe. It is a Very Long Baseline Interferometer (VLBI) system with space-space or space-ground baselines for observations at frequencies above $300 \mathrm{GHz}$ at microarcsecond angular resolution. It is an aperture synthesis system with optimized $u v$-coverage for achieving high imaging fidelity. Origins and THEZA could be seen as highly synergistic pathfinders, where Origins has even higher angular resolution but only a single instantaneous baseline.

The proposal to ESA "Bringing high spatial resolution to the Far-infrared-A giant leap for astrophysics" by Linz et al. [13] suggests either a space-space interferometer or a deployable e.g. ring-like telescope to obtain high spatial resolution to observe, for example, water in proto-planetary discs. This instrument would operate at higher frequencies than THEZA, but at lower spatial resolution to be sensitive to proto-

\footnotetext{
${ }^{3}$ http://millimetron.ru/index.php/en/

${ }^{4}$ https://spica-mission.org/
} 
planetary discs. It would complement Origins water trail observations by being able to image the discs.

There are also smaller missions planned that are pathfinders for Origins both for science and technological developments: e.g., the GUSTO balloon will carry small arrays of heterodyne receivers at $1.4,1.9$, and $4.7 \mathrm{THz}$ to observe the major cooling lines with a $0.8 \mathrm{~m}$ telescope. The SOFIA airplane observatory is equipped with far and mid-IR instruments and is an excellent testbed for technology as well as to advance infrared astronomy. Ground-based interferometers such as ALMA, SMA, or NOEMA interferometers, as well as single dish telescopes such as APEX, ASTE, CCAT prime, and AtLAST complement Origins, as they allow observations at longer wavelengths and at higher spatial resolution. However, due to the atmospheric transmission they cannot operate in the mid or far-IR, which is unique to space.

\section{Origins space telescope (Origins) science and technology definition team (STDT) members}

Margaret Meixner, STScI/JHU, Baltimore, MD (Origins Study Chair). Asantha Cooray, University of California at Irvine, Irvine, CA (Origins Study Chair).

Dave Leisawitz, NASA GSFC, Greenbelt, MD (Origins Study Scientist). Johannes Staguhn, JHU/GSFC, Greenbelt, MD (Origins Deputy Study Scientist). Lee Armus, California Institute of Technology/IPAC, Pasadena, CA.

Cara Battersby, University of Connecticut, Storrs, CT.

James "Gerbs" Bauer, University of Maryland, College Park MD.

Edwin "Ted" Bergin, University of Michigan, Ann Arbor, MI.

Charles "Matt" Bradford, California Institute of Technology/JPL, Pasadena, CA. Kimberly Ennico-Smith, NASA Ames Research Center, Moffett Field, CA.

Jonathan Fortney, University of California, Santa Cruz, CA.

Tiffany Kataria, JPL/NASA, Pasadena, CA.

Gary Melnick, Harvard Smithsonian, Boston, MA.

Stefanie Milam, NASA GSFC, Greenbelt, MD.

Desika Narayanan, University of Florida, Gainesville, FL.

Deborah Padgett, JPL/NASA, Pasadena, CA.

Klaus Pontoppidan, STScI, Baltimore, MD.

Alexandra Pope, University of Mass, Amherst, MA.

Thomas Roellig, NASA Ames Research Center, Moffett Field, CA.

Karin Sandstrom, University of California at San Diego, in La Jolla, CA.

Kevin Stevenson, STScI, Baltimore, MD.

Kate Su, University of Arizona, Tucson, AZ.

Joaquin Vieira, University of Illinois, Urbana-Champaign, Illinois.

Edward L. "Ned" Wright, UCLA, Los Angeles, CA.

Jonas Zmuidzinas, California Institute of Technology/JPL, Pasadena, CA. 


\section{Ex-Officio (non-voting) members of origins science and technology definition team}

Kartik Sheth, NASA HQ, Program Scientist.

Dominic Benford, NASA HQ, Deputy Program Scientist.

Eric E. Mamajek, NASA JPL/Caltech, ExEP Deputy Program Scientist.

Susan Neff, NASA/GSFC, COR Chief Scientist.

Elvire De Beck, Chalmers Institute of Technology, SNSB Liaison.

Maryvonne Gerin, LERMA, Obs. De Paris, CNES Liason.

Frank Helmich, SRON, Netherlands Institute for Space Research Liaison.

Itsuki Sakon, University of Tokyo, JAXA Liaison, MISC instrument lead.

Douglas Scott, University of British Columbia, CAS Liaison.

Roland Vavrek, ESA/ESAC, ESA Liaison.

Martina Wiedner, LERMA, Obs. De Paris, HERO instrument lead.

Sean Carey, Caltech/IPAC, Communications Scientist.

Denis Burgarella, Laboratoire d'Astrophysique de Marseille, collaborator.

Samuel Harvey Moseley, NASA/GSFC, instrument scientist.

Acknowledgements The study was largely funded by National Aeronautics and Space Administration (NASA), which allowed us to hire engineering staff, subcontract parts of the study to industry and to pay for travel and meetings. The non-American team members are grateful for funding from their respective space agency and/or their research institute, in particular Centre National de Recherche Scientifiques (CNES), Swedish National Space Agency, Netherlands Institute for Space Research (SRON), Japan Aerospace Exploration Agency (JAXA).A large team of people carried out the Origins Space Telescope study. The Science and Technology Definitions Team (consisting of 25 American voting members, and 14 international non-voting members) defined the satellite in open meetings with additional interested scientists joining in the discussions, and the NASA/GSFC Study Center Engineering Design team carried out a preliminary design. There were also US industry studies and advice, as well as a Study Advisory Board. Margaret Meixner and Asantha Cooray are the joint P.I.s of the study.(Martina Wiedner is the instrument lead of the mostly European HERO study and design.)

Open Access This article is licensed under a Creative Commons Attribution 4.0 International License, which permits use, sharing, adaptation, distribution and reproduction in any medium or format, as long as you give appropriate credit to the original author(s) and the source, provide a link to the Creative Commons licence, and indicate if changes were made. The images or other third party material in this article are included in the article's Creative Commons licence, unless indicated otherwise in a credit line to the material. If material is not included in the article's Creative Commons licence and your intended use is not permitted by statutory regulation or exceeds the permitted use, you will need to obtain permission directly from the copyright holder. To view a copy of this licence, visit http://creativecommons.org/licenses/by/4.0/.

\section{References}

1. Bolatto, A. et al. 2019.: "Cold Gas Outflows, Feedback, and the Shaping of Galaxies," US Science White Paper.

2. Boogert, A.C.A., Gerakines, P.A., Whittet, D.C.B.: Observations of the icy Universe. Ann. Rev. Astron. Astrophys. 53, 541 (2015) 
3. Caselli, P., et al.: First Detection of Water Vapor in a Pre-stellar Core. The Astrophysical Journal Letters 759(2), L37 (2012)

4. Delabrouille, J., Abitbol, M. H., Aghanim, N., Ali-Haimoud, Y., Alonso, D., Alvarez, M, B., Anthony J., Bartlett, J. G., Baselmans, J, Basu, K., Battaglia, N., Bermejo C., Jose R., Bernal, J. L., Béthermin, M., Bolliet, B., Bonato, M., Bouchet, F. R., Breysse, P. C., Burigana, C., Cai, Z. Y., Chluba, J., Churazov, E., Dannerbauer, H., De Bernardis, P., De Zotti, G., Di Valentino, E. Dimastrogiovanni, E., Endo, A., Erler, J., Ferraro, S., Finelli, F., Fixsen, D., Hanany, S., Hart, L., Hernandez-Monteagudo, C., Hill, J., Colin, H., Selim C., Karatsu, K., Karkare, K., Keating, G. K., Khabibullin, I., Kogut, A., Kohri, K., Kovetz, E. D., Lagache, G., Lesgourgues, J., Madhavacheril, M., Maffei, B., Mandolesi, N., Martins, C., Masi, S., Mather, J. M., Jean-Baptiste, M., Dizgah, A., Mroczkowski, T., Mukherjee, S., Nagai, D., Negrello, M., Palanque-Delabrouille, N., Paoletti, D., Patil, S. P., Piacentini, F., Raghunathan, S., Ravenni, A., Remazeilles, M., Revéret, V., Rodriguez, L., Rotti, A., Rubino M., Jose-Alberto, S, J., Scott, D., Silk, J., Silva, M., Souradeep, T., Sugiyama, N., Sunyaev, R., Switzer, E. R., Tartari, A, Trombetti, T., Zubeldia, I. "Microwave Spectro-Polarimetry of Matter and Radiation across Space and Time", Experimental Astronomy, 2021, this issue

5. Hartogh, P., Lis, D. C., Bockelée-Morvan, D., de Val-Borro, M., Biver, N., Küppers, M., Emprechtinger, M., Bergin, E. A., Crovisier, J., Rengel, M ., Moreno, R., Szutowicz, S. and Blake, G. A. "Ocean-like water in the Jupiter-family comet 103P/Hartley 2," Nature, 478, 218-220, https://doi.org/10.1038/ nature 10519, 2011a

6. Hartogh, P., Lellouch, E., Moreno, R., Bockelée-Morvan, D.,Biver, N.,Cassidy, T. Rengel, M., Jarchow, C., Cavalié, J. Crovisier, T., Helmich, F. P. and Kidger, M. "Direct detection of the Enceladus water torus with Herschel”, Astron. \& Astrophys., 532, L2, https://doi.org/10.1051/0004-6361/201117377, 2011 b

7. Gurvits L.I., Paragi Z., Casasola V., Conway J.E., Davelaar J., Falcke H., Fender R., Frey S., Fromm Ch.M., García Miró C., Garrett M.A., Giroletti M., Goddi C., Gómez J.-L., van der Gucht J., Guirado J.C., Haiman Z., Helmich F., Humphreys E., Impellizzeri V., Kramer M., Lindqvist M., Linz H., Liuzzo E., Lobanov A.P., Mizuno Y., Rezzolla L., Roelofs F., Ros E., Rygl K.L.J., Savolainen T., Schuster K., Venturi T., Wiedner M., Zensus J.A, 2021, "TeraHertz Exploration and Zooming-in for Astrophysics (THEZA): ESA Voyage 2050 White Paper", Experimental Astronomy, this issue

8. Kataria, T. et al. 2019, "The Mid-Infrared Search for Biosignatures on Temperate M-Dwarf Planets", US Science White Paper no 462

9. Keto, E., Rawlings, J., Caselli, P.: Chemistry and radiative transfer of water in cold, dense clouds. Mon. Not. R. Astron. Soc. 440(3), 2614 (2014)

10. Keto, E., Rawlings, J., Caselli, P.: The dynamics of collapsing cores and star formation. Mon. Not. R. Astron. Soc. 446(4), 3731 (2015)

11. Küppers, M., O’Rourke, L., Bockelee-Morvan, D., Zakharov, V., Seungwon, L., von Allmen, P., Carry, B., Teyssier, D., Marston, A., Müller, T., Crovisier, J., Barucci, M.A., Moreno, R. "Localized sources of water vapour on the dwarf planet (1)Ceres," Nature, 505, 525-527, https://doi.org/10.1038/nature12918, 2014

12. Line, M., Quanz, S. P., Schwieterman, E. W., Fortney, J. J., Stevenson, K. B., Greene, T., Zellem, R., Morley, C., Kataria, T., Tremblay, L., Mennesson, B, Iyer, A., Mawet, D., Iro, N., Kaltenegger, L., Defrere, D., Kite, E. S., Caldwell, D. A., Shkolnik, E., Dragomir, D., Ngo, H., Blecic, J., Lichtenberg, T., Angerhausen, D., Solmaz, A., Wolf, E. T., Monnier, J., Hicks, B., Kane, S. R., Danchi, W., Stassun, K., Valencia, D., Staguhn, J., "The Importance of Thermal Emission Spectroscopy for Understanding Terrestrial Exoplanets" Astro2020: Decadal Survey on Astronomy and Astrophysics, science white papers, no. 271, Bulletin of the American Astronomical Society, Vol. 51, Issue 3, id. 271, 2019

13. Linz, H., Beuther, H., Gerin, M., Goicoechea, J.R., Helmich, F., Krause, O., Liu, Y., Molinari, S., Ossenkopf-Okada, V., Pineda, J., Sauvage, M., Schinnerer, E., van der Tak, F., Wiedner, M., Amiaux, J., Bhatia, D., Buinhas, L., Durand, G., Förstner, R., Graf, U., Lezius, M., "Bringing high spatial resolution to the Far-infrared - A giant leap for astrophysics", Experimental Astrophysics, 2021, this issue

14. Leisawitz, D., Amatucci, E., Allen, L., Arenberg, J., Armus, L., Battersby, C., Bauer, J., Beaman, B.G., Bell, R., Beltran, P., Benford, D., Bergin, E., Bolognese, J., Bradford, C.M., Bradley, D., Burgarella, D., Carey, S., Carter, R., Chi, J. D., Cooray, A., Corsetti, J., D’Asto, T., De Beck, E., Denis, K., Derkacz, C., Dewell, L., DiPirro, M., Earle, C.P., East, M., Edgington, S., Ennico, K., Fantano, L., Feller, G., Folta, D., Fortney, J., Gavares, B.J., Generie, J., Gerin, M., Granger, Z., Greene, T.P., Griffiths, A., Harpole, G., Harvey, K., Helmich, F. P., Hilliard, L., Howard, J., Jacoby, M., Jamil, A., Jamison, T., Kaltenegger, L., Kataria, T., S. Knight, J.S., Knollenberg, P., Lawrence, C., Lightsey, P., Lipscy, S., Mamajek, E., Martins, G., Mather, J.C., Meixner, M., Melnick, G., Milam, S., Mooney, T., Moseley, S.H., Narayanan, D., Neff, S., Nguyen, T., Nordt, A., Olson, J., Padgett, D., Petach, M., Petro, S., Pohner, J., Pontoppidan, K., Pope, A., Ramspacker, D., Rao, A., Roellig, T., Sakon, I., Sandin, C., Sandstrom, K., 
Scott, D., Seals, L., Sheth, K., Sokolsky, L.M., Staguhn, J., Steeves, J., Stevenson, K., Stoneking, E., Su, K., Tajdaran, K., Tompkins, S., Vieira, J., Webster, C., Wiedner, M.C., Wright, E.L., Wu, C., Zmuidzinas, J. "The Origins Space Telescope: baseline mission concept," J. Astron. Telesc. Instrum. Syst. 7(1), 011002 (2021), https://doi.org/10.1117/1.JATIS.7.1.011002.

15. Meixner, M. et al. "Origins Space Telescope Science Drivers to Design Traceability", J. Astron. Telesc. Instrum. Syst., accepted 2021

16. Moreno, E. Lellouch, L. Lara, H. Feuchtgruber, M. Rengel, P. Hartogh, and R. Courtin, "The abundance, vertical distribution and origin of $\mathrm{H} 2 \mathrm{O}$ in Titan's atmosphere: Herschel observations and photochemical modeling", Icarus, 221, 753-767, https://doi.org/10.1016/j.icarus.2012.09.006, 2012.

17. Öberg, K.I., Murray-Clay, R., Bergin, E.A.: The Effects of Snowlines on C/O in Planetary Atmospheres. The Astrophysical Journal Letters 743, L16 (2011)

18. Pesce, D. P., Haworth, K., Melnick, Gary J., Blackburn, L., Wielgus, M., Johnson, M. D., Raymond, A., Weintroub, J., Palumbo, D. C. M., Doeleman, S. S., James, D. J., " Extremely long baseline interferometry with Origins Space Telescope", Astro2020: Decadal Survey on Astronomy and Astrophysics, APC white papers, no. 176, Bulletin of the American Astronomical Society, Vol. 51, Issue 7, id. 176 (2019).

19. Pope, A. et al. 2019, "Simultaneous Measurements of Star Formation and Supermassive Black Hole Growth in Galaxies", US Decadal White Paper.

20. Rigopoulou, D., Pearson, C. , Ellison, B., Wiedner, M. C. , Ossenkopf Okada, V., Kok Tan, B., Garcia Bernete, I., Gerin, M., Caux, E., Molinari, S., Goicoechea, J. R., Lis, D. L., Goldsmith, P. F., Aalto, S., Magdis, G., Kramer, C. "The Far-Infrared Spectroscopic Surveyor (FIRSS)" Experimental Astrophysics, 2021, this issue

21. Sadavoy, S., et al.: The Life Cycle of Dust. BAAS 51, 66 (2019)

22. Shaposhnikov, D.S., Medvedev, A.S., Rodin, A.V., Hartogh, P.: Seasonal Water “'Pump' in the Atmosphere of Mars: Vertical Transport to the Thermosphere.” Geophys. Res. Lett. 46(8), 4161-4169 (2019)

23. Smith, J.D., et al.: The Chemical Enrichment History of the Universe. BAAS 51, 400 (2019)

24. Villanueva, L., Mumma, M. J., Novak, R. E., Käufl, H. U., Hartogh, P., Encrenaz, T., Tokunaga, A., Khayat, A., and Smith, M. D. "Strong water isotopic anomalies in the martian atmosphere: Probing current and ancient reservoirs", Science, 348, aaa3630, https://doi.org/10.1126/science.aaa3630, 2015.

25. Whittet, D.C.B., et al.: Interstellar ice grains in the Taurus molecular clouds. Nature 303, 218 (1983)

26. Wiedner, M.C., Aalto, S., Amatucci, E.G., Baryshev, A., Battersby, C., Belitsky, V., Bergin, E.A., Borgo, B., Carter, R.C., Caux, E., Cooray, A., Corsetti, J.A., De Beck, E., Delorme, Y., Desmaris, V., Dipirro, M.J., Ellison, B., Di Giorgio, A.M., Eggens, M., Gallego, J.-D., Gerin, M., Goldsmith, P.F., Goldstein, C., Helmich, F., Herpin, F., Hills, R.E., Hogerheijde, M.R., Hunt, L.K., Jellema, W., Keizer, G., Krieg, J.-M., Kroes, G., Laporte, P., Laurens, A., Leisawitz, D.T., Lis, D.C., Martins, G.C., Mehdi, I., Meixner, M., Melnick, G., Milam, S.N., Neufeld, D.A., Nguyen Tuong, N., Plume, R., Pontoppidan, K.M., Quertier-Dagorn, B., Risacher, C., Staguhn, J.G., Tong, E., Viti, S., Wyrowski, F.: Heterodyne Receiver for Origins. J. Astron. Telesc. Instrum. Syst. 7(1), 011007 (2021). https://doi.org/10.1117/1. JATIS.7.1.011007

Publisher's Note Springer Nature remains neutral with regard to jurisdictional claims in published maps and institutional affiliations.

\section{Affiliations}

M. C. Wiedner ${ }^{1}$ - S. Aalto ${ }^{2}$ - L. Armus ${ }^{3}$ - E. Bergin ${ }^{4}$ - J. Birkby ${ }^{5}$ - C. M. Bradford ${ }^{6}$. D. Burgarella ${ }^{7}$ - P. Caselli ${ }^{8}$ • V. Charmandaris ${ }^{9}$ - A. Cooray ${ }^{10}$ - E. De Beck ${ }^{2}$. J. M. Desert $^{5} \cdot$ M. Gerin ${ }^{1}$ - J. Goicoechea ${ }^{11}$ - M. Griffin ${ }^{12}$ - P. Hartogh ${ }^{13} \cdot$ F. Helmich $^{14}$.

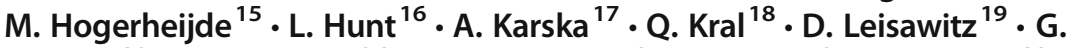

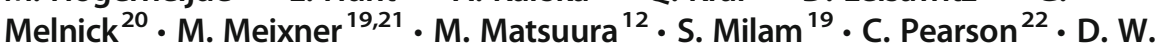
Pesce $^{20}$ - K. M. Pontoppidan 21 - A. Pope ${ }^{23}$ - D. Rigopoulou ${ }^{24}$ - T. Roellig ${ }^{25}$ • I. Sakon $^{26} \cdot$ J. Staguhn ${ }^{19} \cdot$ K. Stevenson ${ }^{21}$

1 Observatoire de Paris, PSL University, CNRS, LERMA, Sorbonne Université, Paris, France

2 Chalmers University of Technology, Gothenburg, Sweden 
IPAC, California Institute of Technology, Pasadena, CA, USA

4 University of Michigan, Ann Arbor, USA

5 University of Amsterdam, Amsterdam, The Netherlands

6 JPL/Caltech, Pasadena, CA, USA

7 University Laboratoire d Astrophysique de Marseille, Marseille, France

8 MPI for for Extraterrestrial Physics, Garching, Germany

9 University of Crete, Crete, Greece

10 UoC At Irvine, Irvine, USA

11 CSIC, Madrid, Spain

12 Cardiff University, Cardiff, UK

13 MPI for Solar System Research, Goettingen, Germany

14 SRON \& Univ of Groningen, Groningen, The Netherlands

15 Leiden University, Leiden, The Netherlands

16 Observatorio Astrofisico Di Arcetri, Florence, Italy

17 Nicolaus Copernicus University, Torun, Poland

18 LESIA, Observatoire de Paris, Université PSL, CNRS, Sorbonne Université, Univ. Paris Diderot, Paris, France

19 NASA/GSFC, Greenbelt, USA

20 Center for Astrophysics, Harvard \& Smithsonian, Cambridge, USA

21 Space Telescope Science Institute, Baltimore, USA

22 Rutherford Appleton Laboratory, Didcot, UK

23 Unveristy of Massachusetts Amerherst, Amherst, MA, USA

24 University of Oxford, Oxford, UK

25 NASA/Ames, Ames, USA

26 University of Tokyo, Tokyo, Japan 